TRANSACTIONS OF THE

AMERICAN MATHEMATICAL SOCIETY

Volume 356, Number 9, Pages 3405-3428

S 0002-9947(04)03592-5

Article electronically published on April 26, 2004

\title{
COMPLETE LINEAR WEINGARTEN SURFACES OF BRYANT TYPE. A PLATEAU PROBLEM AT INFINITY
}

\author{
JOSÉ ANTONIO GÁLVEZ, ANTONIO MARTÍNEZ, AND FRANCISCO MILÁN
}

\begin{abstract}
In this paper we study a large class of Weingarten surfaces which includes the constant mean curvature one surfaces and flat surfaces in the hyperbolic 3 -space. We show that these surfaces can be parametrized by holomorphic data like minimal surfaces in the Euclidean 3-space and we use it to study their completeness. We also establish some existence and uniqueness theorems by studing the Plateau problem at infinity: when is a given curve on the ideal boundary the asymptotic boundary of a complete surface in our family? and, how many embedded solutions are there?
\end{abstract}

\section{INTRODUCTION}

During the last two decades there has been a renewed interest in the geometry of surfaces in hyperbolic manifolds. Basic problems such as existence, symmetry, behaviour at infinity, global structure and classification of complete surfaces of constant curvature in the hyperbolic 3 -space $\mathbb{H}^{3}$ have called the attention of several authors (see, for example, [5], [11, 12], 18]).

Concerning constant mean curvature one surfaces in $\mathbb{H}^{3}$, also called Bryant surfaces, it was crucial the paper of R. Bryant [2, who showed how to parametrize these surfaces by meromorphic data and began the study of their geometry. Since Bryant's work many properties and examples have been discovered in 3], [15], 22], [25], [26] among others. By considering the complex structure determined by the second fundamental form, the authors proved in [6] that a "holomorphic resolution" like the Bryant representation also holds for flat surfaces in $\mathbb{H}^{3}$ and used it in the study of some examples and the behaviour at infinity of complete ends. The most important tool in these representations is that both kinds of surfaces have a conformal hyperbolic Gauss map with respect to a natural complex structure determined by either the first, $I$, or the second, $I I$, fundmental form.

In this paper we extend the two above-mentioned Weierstrass-type representations through the investigation of Weingarten surfaces. We shall study surfaces in $\mathbb{H}^{3}$ whose mean curvature $H$ and Gauss curvature $K_{I}$ satisfy a linear relation of the form

$$
2 a(H-1)+b K_{I}=0
$$

Received by the editors November 11, 2002.

2000 Mathematics Subject Classification. Primary 53C42; Secondary 53A35.

Key words and phrases. Hyperbolic 3-space, Weingarten surfaces, Plateau problem, Weierstrass data.

This research was partially supported by MCYT-FEDER Grant No. BFM2001-3318 and Junta de Andalucía CEC: FQM0804. 


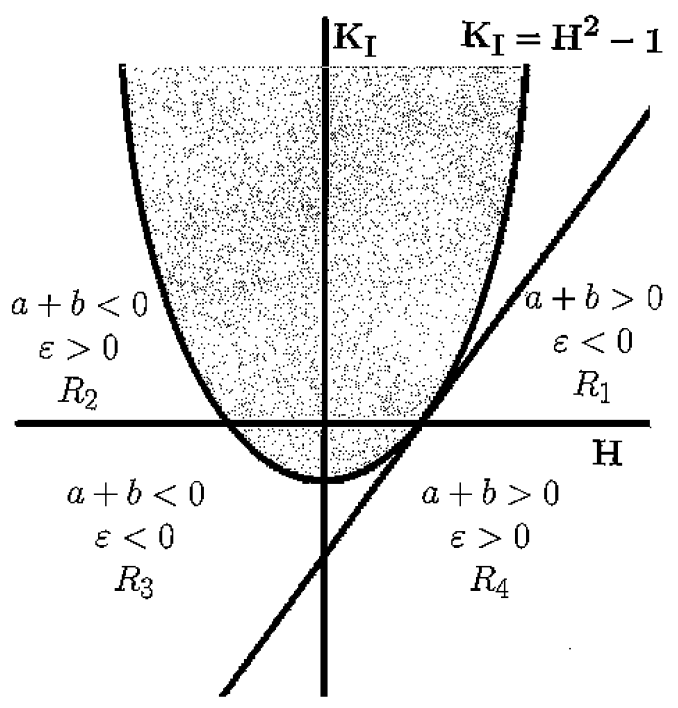

FiguRE 1.

for some $a, b \in \mathbb{R}$, of elliptic type, i.e. $a+b \neq 0$. We call them linear Weingarten surfaces of Bryant type, in short, BLW-surfaces. These surfaces are a particular case of the special surfaces studied by Sa Earp and Toubiana in 21] (see also [17, [19] and [20]).

A BLW-surface $\Sigma$ may be considered lying on a straight half-line $\lambda_{a b}$ in the $\left(H, K_{I}\right)$-plane, starting at the point $(1,0): \lambda_{a b}=\{(1-t b, 2 t a): t \geq 0\}$; see Figure 1 and Remark 3 The geometry of $\Sigma$ is rather different depending on whether $\Sigma$ is in one of the following regions:

$$
\begin{aligned}
& R_{1}=\left\{\left(H, K_{I}\right): 1 \leq H, 0 \leq K_{I}\right\}, \\
& R_{2}=\left\{\left(H, K_{I}\right): 0 \leq K_{I}, 2(H-1) \leq K_{I}\right\}, \\
& R_{3}=\left\{\left(H, K_{I}\right): 2(H-1) \leq K_{I} \leq 0\right\}, \\
& R_{4}=\left\{\left(H, K_{I}\right): 2(H-1) \geq K_{I}, 0 \geq K_{I}\right\} .
\end{aligned}
$$

If $\Sigma$ is in $\lambda_{a b}$, we shall see that

$$
\sigma=a I+b I I
$$

is a Riemannian metric which induces a natural complex structure on $\Sigma$ such that its hyperbolic Gauss map is a conformal map. We shall prove that the complex representations of Bryant [2] and the authors [6] extend to BLW-surfaces (see Theorem 2).

The previous complex resolution is applied to the study of completeness. Thus, we shall start by proving (Theorem 3) that a complete BLW-surface in $R_{1} \cup R_{2}$ is a totally umbilical round sphere, a horosphere or a hyperbolic cylinder.

The regions $R_{3}$ and $R_{4}$ are much richer in global examples. Every complete BLW-surface $\Sigma$ in $\lambda_{a b} \subset R_{4}$, with meromorphic data $(h, \omega)$ has an associated complete constant mean curvature one surface, $\Sigma_{0}$, with geometry as $\Sigma$ and with meromorphic data $(\sqrt{a /(a+b)} h, \omega)$. 
Concerning $R_{3}$, we shall see that complete BLW-surfaces in $\lambda_{a b} \subset R_{3}$ are conformally equivalent to the unit disk, $\mathbb{D}$, and we shall prove (Theorem 4 ) that they are, up to conformal transformations of $\mathbb{D}$, in correspondence with the set of meromorphic maps $G: \mathbb{D} \longrightarrow \mathbb{C} \cup\{\infty\}$ with bounded Schwarzian derivative. In that way the hyperbolic Gauss map and the standard Euclidean Gauss map of complete BLW-surfaces in the half space model of $\mathbb{H}^{3}$ lying on $R_{3}$ give geometric meaning to classical families of complex functions which have been studied in connection with the Schwarzian derivative (see, for example, [9], 13], 14], [16]).

We also establish some existence and uniqueness theorems for complete BLWsurfaces in $R_{3}$. Our main interest shall be the Plateau problem at infinity: when is a given curve on the ideal boundary $\mathbb{S}_{\infty}^{2}$ of $\mathbb{H}^{3}$ the asymptotic boundary of a complete BLW-surface?

If $\lambda_{a b} \subset R_{3}$, we shall prove (Theorem [5) that a convex Jordan curve $\Gamma \subset \mathbb{S}_{\infty}^{2}$ is the asymptotic boundary of a unique embedded complete BLW-surface in $\lambda_{a b}$ with an "inner" hyperbolic normal. Moreover if $-1 / 2<a /(a+b)<0$ there are exactly two embedded complete BLW-surfaces on $\lambda_{a b}$ with asymptotic boundary $\Gamma$.

Finally, we shall deal with the general case and shall prove (Theorem [6) that if $-1 / 2<a /(a+b)<0$, a Jordan curve $\Gamma$ on $\mathbb{S}_{\infty}^{2}$ is the asymptotic boundary of at least two complete BLW-surfaces on $\lambda_{a b}$. If $-1 / 4<a /(a+b)<0$, then there only exist two embedded complete BLW-surfaces on $\lambda_{a b}$ with asymptotic boundary $\Gamma$.

\section{Conformal Representation}

In the Lorentz-Minkowski model, $\mathbb{L}^{4}$, the hyperbolic 3 -space is described by one connected component of a two-sheeted hyperboloid. More precisely,

$$
\mathbb{H}^{3}=\left\{\left(x_{0}, x_{1}, x_{2}, x_{3}\right) \in \mathbb{R}^{4}:-x_{0}^{2}+x_{1}^{2}+x_{2}^{2}+x_{3}^{2}=-1, x_{0}>0\right\}
$$

endowed with the induced metric of $\mathbb{L}^{4}$ given by the quadratic form $-x_{0}^{2}+x_{1}^{2}+$ $x_{2}^{2}+x_{3}^{2}$.

Associated with that metric we shall denote by $\wedge$ the usual exterior product in $\mathbb{H}^{3}$, that is,

$$
u \wedge v=p \times u \times v,
$$

for any tangent vectors $u, v$ at a point $p \in \mathbb{H}^{3}$, where $p \times u \times v$ is the unique vector in $\mathbb{L}^{4}$ such that $\langle p \times u \times v, w\rangle=\operatorname{det}(p, u, v, w)$, for any $w \in \mathbb{L}^{4}$. Here $\langle.,$.$\rangle denotes$ the inner product in $\mathbb{L}^{4}$ and det the usual determinant.

If we consider

$$
\mathbb{N}_{+}^{3}=\left\{\left(x_{0}, x_{1}, x_{2}, x_{3}\right) \in \mathbb{R}^{4}:-x_{0}^{2}+x_{1}^{2}+x_{2}^{2}+x_{3}^{2}=0, x_{0}>0\right\}
$$

the positive null cone in $\mathbb{L}^{4}$, then $\mathbb{N}_{+}^{3} / \mathbb{R}^{+}$inherits a natural conformal structure and it can be regarded as the ideal boundary $\mathbb{S}_{\infty}^{2}$ of $\mathbb{H}^{3}$.

We shall regard $\mathbb{L}^{4}$ as the space of $2 \times 2$ Hermitian matrices, $\operatorname{Herm}(2)$, in the standard way (see [2], [6]), by identifying $\left(x_{0}, x_{1}, x_{2}, x_{3}\right) \in \mathbb{L}^{4}$ with the matrix

$$
\left(\begin{array}{cc}
x_{0}-x_{3} & x_{1}+i x_{2} \\
x_{1}-i x_{2} & x_{0}+x_{3}
\end{array}\right) \text {. }
$$

Under this identification one has $\langle m, m\rangle=-\operatorname{det}(m)$ for all $m \in \operatorname{Herm}(2)$. Then $\mathbb{H}^{3}$ is the set of $m \in \operatorname{Herm}(2)$ with $\operatorname{det}(m)=1$. The action of $\mathbb{S L}(2, \mathbb{C})$ on these Hermitian matrices defined by

$$
g \cdot m=g m g^{*},
$$


where $g \in \mathbb{S L}(2, \mathbb{C}), g^{*}={ }^{t} \bar{g}$, preserves the inner product and leaves $\mathbb{H}^{3}$ invariant.

The space $\mathbb{N}_{+}^{3}$ is seen as the space of positive semi-definite $2 \times 2$ Hermitian matrices of determinant 0 and its elements can be written as $w^{t} \bar{w}$, where ${ }^{t} w=\left(w_{1}, w_{2}\right)$ is a nonzero vector in $\mathbb{C}^{2}$ uniquely defined up to multiplication by a unimodular complex number. The map $w^{t} \bar{w} \longrightarrow\left[\left(w_{1}, w_{2}\right)\right] \in \mathbb{C P}^{1}$ becomes the quotient map of $\mathbb{N}_{+}^{3}$ on $\mathbb{S}_{\infty}^{2}$ and identifies $\mathbb{S}_{\infty}^{2}$ to $\mathbb{C P}^{1}$. Thus, the natural action of $\mathbb{S L}(2, \mathbb{C})$ on $\mathbb{S}_{\infty}^{2}$ is the action of $\mathbb{S L}(2, \mathbb{C})$ on $\mathbb{C P}^{1}$ by Möbius transformations.

Now, let $S$ be a surface and $\psi: S \longrightarrow \mathbb{H}^{3}$ an immersion with Gauss map $\eta$. Then, $\psi$ is called a linear Weingarten immersion of Bryant type (in short, BLW-surface) if the mean curvature $H$ and the Gauss curvature $K_{I}$ satisfy a linear relation of the form

$$
2 a(H-1)+b K_{I}=0
$$

for some $a, b \in \mathbb{R}, a+b \neq 0$.

Remark 1. This particular kind of linear Weingarten immersion includes the Bryant surfaces and the flat surfaces.

The immersions satisfying (2) with $a+b=0$, that is, the nonelliptic case, are the ones with a constant principal curvature equal to 1 . They are studied in [1].

Lemma 1. Let $\psi: S \longrightarrow \mathbb{H}^{3}$ be a BLW-surface. Then, we can consider that $|a+b|=$ 1 ,

$$
2 a(H-1)+b(K-1)=0
$$

and $\sigma=a I+b I I$ is a positive definite metric, where $K$ is the Gauss-Kronecker curvature, $I=\langle d \psi, d \psi\rangle$ and $I I=\langle d \psi,-d \eta\rangle$, the first and second fundamental form of the immersion, respectively.

Proof. Since $K_{I}=K-1$, by scaling $a$ and $b$ in (2), we can assume that 3) is satisfied, with $a+b=1$.

Let $\left\{e_{1}, e_{2}\right\}$ be an orthonormal basis at a point $p$ which diagonalizes $d \eta$, that is, $d \eta\left(e_{i}\right)=-k_{i} e_{i}, i=1,2$. Then,

$$
\begin{gathered}
\sigma\left(e_{1}, e_{1}\right) \sigma\left(e_{2}, e_{2}\right)-\sigma\left(e_{1}, e_{2}\right)^{2}=\left(a+b k_{1}\right)\left(a+b k_{2}\right) \\
=a^{2}+b(2 a H+b K)=(a+b)^{2}=1
\end{gathered}
$$

and so $\sigma$ is definite. Now, by changing the sign of $a$ and $b$ if necessary, we obtain that $\sigma$ is positive definite and $|a+b|=1$, which concludes the proof.

From now on, we will suppose that every BLW-surface satisfies the above lemma and we shall regard $S$ as a Riemann surface with the conformal structure induced by $\sigma=a I+b I I$.

Lemma 2. Let $\psi: S \longrightarrow \mathbb{H}^{3}$ be a BLW-surface and $z$ be a conformal parameter for $\sigma$. Then

$$
a \psi_{z}-b \eta_{z}=-i \eta \wedge \psi_{z}
$$

where, for instance, $\psi_{z}$ denotes the derivative of $\psi$ with respect to $z$.

Proof. Let $z$ be a conformal parameter such that $\sigma=2 \rho|d z|^{2}$. From (4),

$$
\left\langle\psi_{z} \wedge \psi_{\bar{z}}, \eta\right\rangle=i \rho .
$$


Since $a \psi_{z}-b \eta_{z}$ is a tangent vector field, it can be written as

$$
a \psi_{z}-b \eta_{z}=\alpha \eta \wedge \psi_{z}+\beta \eta \wedge \psi_{\bar{z}}
$$

for some local complex functions $\alpha$ and $\beta$.

Now, using that $z$ is conformal and (6),

$$
\begin{aligned}
& 0=\sigma\left(\psi_{z}, \psi_{z}\right)=\left\langle a \psi_{z}-b \eta_{z}, \psi_{z}\right\rangle=\beta\left\langle\eta \wedge \psi_{\bar{z}}, \psi_{z}\right\rangle=-i \beta \rho, \\
& \rho=\sigma\left(\psi_{z}, \psi_{\bar{z}}\right)=\left\langle a \psi_{z}-b \eta_{z}, \psi_{\bar{z}}\right\rangle=\alpha\left\langle\eta \wedge \psi_{z}, \psi_{\bar{z}}\right\rangle=i \alpha \rho
\end{aligned}
$$

and we are done.

As in Bryant's paper [2], we consider for any immersion $\psi: S \longrightarrow \mathbb{H}^{3}$ with associated Gauss map $\eta$ its hyperbolic Gauss map as the map given by $G:=[\psi+\eta]$. That is, for any $p \in S$, the oriented geodesic emanating from $p$ meets the ideal boundary $\mathbb{S}_{\infty}^{2}$ of $\mathbb{H}^{3}$ at $G(p)$.

Theorem 1. Let $\psi: S \longrightarrow \mathbb{H}^{3}$ be a BLW-surface. Then $\psi+\eta$ is a conformal map with respect to the metric $\sigma=a I+b I I$ and

$$
\Delta^{\sigma}(\psi+\eta)=\frac{2}{a+b}\{(H-1) \psi+(K-H) \eta\},
$$

where $\Delta^{\sigma}$ denotes the Laplacian of $\sigma$. In particular, its hyperbolic Gauss map $G$ is conformal.

Moreover, the immersion lies in a horosphere or $\langle d(\psi+\eta), d(\psi+\eta)\rangle$ is a pseudometric of constant curvature $\frac{a}{a+b}$.

Proof. Let $z$ be a conformal parameter such that $\sigma=2 \rho|d z|^{2}$. Then we can write

$$
\begin{aligned}
\eta_{z} & =-\Lambda \psi_{z}+\Upsilon \psi_{\bar{z}}, \\
\eta_{\bar{z}} & =\bar{\Upsilon} \psi_{z}-\Lambda \psi_{\bar{z}}
\end{aligned}
$$

for a local real function $\Lambda$ and a complex function $\Upsilon$.

Thus, $H=\Lambda$ and $K=\Lambda^{2}-|\Upsilon|^{2}=H^{2}-|\Upsilon|^{2}$, that is,

$$
\eta_{z}=-H \psi_{z}+\Upsilon \psi_{\bar{z}} \text {. }
$$

Therefore, using (3),

$$
(a+b H)^{2}-b^{2}|\Upsilon|^{2}=a^{2}+2 a b H+b^{2} K=(a+b)^{2}=1 .
$$

The cross product of (5) and (8) with $\psi_{z}$ and $\psi_{\bar{z}}$ gives

$$
\begin{aligned}
& \left\{\begin{array}{l}
-b \eta_{z} \wedge \psi_{z}=-i\left(\eta \wedge \psi_{z}\right) \wedge \psi_{z}=i\left\langle\psi_{z}, \psi_{z}\right\rangle \eta \\
-b \eta_{z} \wedge \psi_{z}=-b \Upsilon \psi_{\bar{z}} \wedge \psi_{z}
\end{array}\right. \\
& \left\{\begin{array}{l}
\left(a \psi_{z}-b \eta_{z}\right) \wedge \psi_{\bar{z}}=-i\left(\eta \wedge \psi_{z}\right) \wedge \psi_{\bar{z}}=i\left\langle\psi_{z}, \psi_{\bar{z}}\right\rangle \eta \\
\left(a \psi_{z}-b \eta_{z}\right) \wedge \psi_{\bar{z}}=a \psi_{z} \wedge \psi_{\bar{z}}+b\left(H \psi_{z}-\Upsilon \psi_{\bar{z}}\right) \wedge \psi_{\bar{z}}=(a+b H) \psi_{z} \wedge \psi_{\bar{z}},
\end{array}\right.
\end{aligned}
$$

and by taking the inner product with $\eta$ one has from (6)

$$
\begin{aligned}
\left\langle\psi_{z}, \psi_{z}\right\rangle & =b \Upsilon \rho, \\
\left\langle\psi_{z}, \psi_{\bar{z}}\right\rangle & =(a+b H) \rho .
\end{aligned}
$$

In that way, from (5),

$$
b(\psi+\eta)_{z}=(a+b) \psi_{z}+i \eta \wedge \psi_{z}
$$


and, using (9), one obtains $\left\langle(\psi+\eta)_{z},(\psi+\eta)_{z}\right\rangle=0$ when $b \neq 0$. Moreover, if $b=0$, then, from (31) and (8), we have that $H=1,(\psi+\eta)_{z}=\Upsilon \psi_{\bar{z}}$ and, using (9), $\left\langle(\psi+\eta)_{z},(\psi+\eta)_{z}\right\rangle=0$. Therefore, $\psi+\eta$ is a conformal map in any case.

Now, bearing in mind (10) and (6), if we take the derivative of (5) with respect to $\bar{z}$, then

$$
a \psi_{z \bar{z}}-b \eta_{z \bar{z}}=\rho \psi-i H \psi_{z} \wedge \psi_{\bar{z}}-i \psi \times \eta \times \psi_{z \bar{z}} .
$$

As the above equality has imaginary part $0=\psi \times \eta \times \psi_{z \bar{z}}$, we have

$$
a \psi_{z \bar{z}}-b \eta_{z \bar{z}}=\rho(\psi+H \eta) .
$$

On the other hand, (5) also gives $\left(a \psi_{z}-b \eta_{z}\right) \wedge \eta=-i \psi_{z}$ and by deriving with respect to $\bar{z}$ we obtain

$$
\psi_{z \bar{z}}=\rho\{(a+b H) \psi+(a H+b K) \eta\} .
$$

If $b \neq 0$, then (12) minus (11) (respectively, (12) plus (11)) and (6) give the expression (7) when $a+b=1$ (respectively, when $a+b=-1$ ).

If $b=0$, then $H=1, \sigma=I,\left\langle\psi_{z}, \psi_{z}\right\rangle=0$ and $\left\langle\psi_{z}, \psi_{\bar{z}}\right\rangle=\rho$, hence

$$
\psi_{z z}=\frac{\rho_{z}}{\rho} \psi_{z}-\Upsilon \rho \eta, \quad \psi_{z \bar{z}}=\rho \eta, \quad \eta_{z}=-\psi_{z}+\Upsilon \psi_{\bar{z}} .
$$

Since $\left(\psi_{z z}\right)_{\bar{z}}=\left(\psi_{z \bar{z}}\right)_{z}$, we have $(\rho \Upsilon)_{\bar{z}}=0$ and, consequently,

$$
(\psi+\eta)_{z \bar{z}}=\left(\Upsilon \psi_{\bar{z}}\right)_{\bar{z}}=-|\Upsilon|^{2} \rho \eta=\rho(K-1) \eta .
$$

In order to determine the singular points of $\psi+\eta$, we have from (6), (9) and (10) that

$$
\begin{aligned}
& b^{2}\left\langle(\psi+\eta)_{z},(\psi+\eta)_{\bar{z}}\right\rangle=\left\langle(a+b) \psi_{z}+i \eta \wedge \psi_{z},(a+b) \psi_{\bar{z}}-i \eta \wedge \psi_{\bar{z}}\right\rangle \\
& =2\left(\left\langle\psi_{z}, \psi_{\bar{z}}\right\rangle-(a+b) \rho\right)=2 b(H-1) \rho .
\end{aligned}
$$

We conclude that if $b \neq 0, \psi+\eta$ has a singular point at $z_{0}$ if and only if $H\left(z_{0}\right)=1$. Moreover, from (3) and (9), if $H\left(z_{0}\right)=1$ and $b \neq 0$, then $K\left(z_{0}\right)=1$ and $a+b=1$. When $b=0$ the same conclusion is clear from (8)) (with $a=1$ because $\sigma$ is positive definite).

Now, from (22) and (7), $\psi$ can be written as

$$
\psi=\frac{a+b}{\rho(2 H-K-1)}(\psi+\eta)_{z \bar{z}}+\frac{2 a+b}{2(a+b)}(\psi+\eta),
$$

at the regular points of $\psi+\eta$.

Using that $\psi+\eta$ is conformal, we have that there exist holomorphic functions, $A, B$, such that $[\psi+\eta]$ is represented as $[(A, B)] \in \mathbb{C P}^{1} \equiv \mathbb{S}_{\infty}^{2}$ and

$$
\psi+\eta=\lambda\left(\begin{array}{c}
A \\
B
\end{array}\right)(\bar{A}, \bar{B})=\lambda\left(\begin{array}{cc}
A \bar{A} & A \bar{B} \\
\bar{A} B & B \bar{B}
\end{array}\right),
$$

for some positive regular function $\lambda$. Thus, (14) gives

$$
\psi=g \Omega g^{*} \quad \text { and } \quad \eta=g \widetilde{\Omega} g^{*},
$$

with

$g=\left(\begin{array}{cc}A & A_{z} \\ B & B_{z}\end{array}\right), \Omega=\left(\begin{array}{cc}\delta \lambda_{z \bar{z}}-\beta \lambda & \delta \lambda_{z} \\ \delta \lambda_{\bar{z}} & \delta \lambda\end{array}\right), \widetilde{\Omega}=\left(\begin{array}{cc}(1+\beta) \lambda-\delta \lambda_{z \bar{z}} & -\delta \lambda_{z} \\ -\delta \lambda_{\bar{z}} & -\delta \lambda\end{array}\right)$ 
$\beta=-\frac{2 a+b}{2(a+b)}$ and $\delta=\frac{a+b}{\rho(2 H-K-1)}$. From these expressions one gets

$$
\begin{gathered}
2=\langle\eta, \eta\rangle-\langle\psi, \psi\rangle=-\operatorname{det}(\eta)+\operatorname{det}(\psi)=\delta \lambda^{2}|\operatorname{det}(g)|^{2}, \\
1=-\langle\psi, \psi\rangle=\operatorname{det}(\psi)=-2 \beta+\frac{4}{\lambda^{4}|\operatorname{det}(g)|^{2}}\left(\lambda \lambda_{z \bar{z}}-\lambda_{z} \lambda_{\bar{z}}\right)
\end{gathered}
$$

and, consequently,

$$
\left(\log \left(\lambda^{2}|\operatorname{det}(g)|^{2}\right)\right)_{z \bar{z}}=\frac{1+2 \beta}{2} \lambda^{2}|\operatorname{det}(g)|^{2}=\frac{-a}{2(a+b)} \lambda^{2}|\operatorname{det}(g)|^{2} .
$$

That is, $\langle d(\psi+\eta), d(\psi+\eta)\rangle=\lambda^{2}|\operatorname{det}(g)|^{2}|d z|^{2}$ has constant Gauss curvature $\varepsilon=\frac{a}{a+b}$ at the regular points of $\psi+\eta$.

From (7) and (12), the two 2-forms $Q_{I}=\left\langle\psi_{z}, \psi_{z}\right\rangle d z^{2}$ and $Q_{I I}=\left\langle\psi_{z},-\eta_{z}\right\rangle d z^{2}$ are holomorphic on $S$. And, since $\psi$ has an umbilical point at $z_{0}$ if and only if $Q_{I}\left(z_{0}\right)=0=Q_{I I}\left(z_{0}\right)$, then $\psi$ is totally umbilical or has isolated umbilical points. Hence, either $\psi$ is totally umbilical with $H=1=K$, that is, $\psi(S)$ lies on a horosphere, or $\langle d(\psi+\eta), d(\psi+\eta)\rangle$ is a pseudometric of constant curvature $\varepsilon$.

Remark 2. If $\psi: S \longrightarrow \mathbb{H}^{3}$ is a BLW-surface and $S$ is a topological sphere, then, using the fact that $Q_{I}$ and $Q_{I I}$ are holomorphic, they vanish identically and we obtain that $\psi(S)$ is a totally umbilical round sphere.

Remark 3 . The regions $R_{1}, R_{2}, R_{3}$ and $R_{4}$ given in the Introduction separate into four parts the exterior of the domain $H^{2}-\left(K_{I}+1\right)<0$ in the $\left(H, K_{I}\right)$-plane according to the sign of $a+b$ and $\varepsilon=a /(a+b)$; see Figure 1 .

In fact, if $b \neq 0$, from (7) and (13) we obtain

$$
\frac{2}{b}(H-1)=\frac{1}{\rho}\left\langle(\psi+\eta)_{z},(\psi+\eta)_{\bar{z}}\right\rangle=-\frac{1}{\rho}\left\langle(\psi+\eta)_{z \bar{z}}, \psi+\eta\right\rangle=\frac{2(H-1)-K_{I}}{a+b} .
$$

When $a+b=1$, from (3),

$$
2(H-1) \geq K_{I}=(a+b) K_{I}=a K_{I}-2 a(H-1)=a\left(K_{I}-2(H-1)\right),
$$

that is,

1. if $a \geq 0$, then $2(H-1) \geq K_{I}, K_{I} \leq 0$ and we are in the region $R_{4}$,

2. if $a \leq 0$, then $2(H-1) \geq K_{I} \geq 0$ and we are in the region $R_{1}$.

In a similar way, when $a+b=-1$, we are either in $R_{2}$ or in $R_{3}$.

Moreover, from (13), if $b \geq 0$, it is satisfied that $H \geq 1$ and if $b \leq 0$, then $H \leq 1$. Consequently, from (3), if $a \geq 0$, one gets $K_{I} \leq 0$ and if $a \leq 0$, then $K_{I} \geq 0$.

Theorem 2 (Conformal representation). i) Let $S$ be a noncompact, simply connected surface and $\psi: S \longrightarrow \mathbb{H}^{3}$ a BLW-surface. Then, there exist a meromorphic curve $g: S \longrightarrow \mathbb{S L}(2, \mathbb{C})$ and a pair $(h, \omega)$ consisting of a meromorphic function $h$ and a holomorphic 1-form $\omega$ on $S$, such that the immersion and its Gauss map can be recovered as

$$
\psi=g \Omega g^{*} \quad \text { and } \quad \eta=g \widetilde{\Omega} g^{*}
$$

where

$$
\Omega=\left(\begin{array}{cc}
\frac{1+\varepsilon^{2}|h|^{2}}{1+\varepsilon|h|^{2}} & -\varepsilon \bar{h} \\
-\varepsilon h & 1+\varepsilon|h|^{2}
\end{array}\right) \quad \text { and } \quad \widetilde{\Omega}=\left(\begin{array}{cc}
\frac{1-\varepsilon^{2}|h|^{2}}{1+\varepsilon|h|^{2}} & \varepsilon \bar{h} \\
\varepsilon h & -1-\varepsilon|h|^{2}
\end{array}\right) \text {, }
$$


with $\varepsilon=\frac{a}{a+b}$ and $1+\varepsilon|h|^{2}>0$. Moreover, the curve $g$ satisfies

$$
g^{-1} d g=\left(\begin{array}{cc}
0 & \omega \\
d h & 0
\end{array}\right) .
$$

The induced metric and $\sigma=a I+b I I$ are given, respectively, by

$$
I=(1-\varepsilon) \omega d h+\left(\frac{(1-\varepsilon)^{2}|d h|^{2}}{\left(1+\varepsilon|h|^{2}\right)^{2}}+\left(1+\varepsilon|h|^{2}\right)^{2}|\omega|^{2}\right)+(1-\varepsilon) \bar{\omega} d \bar{h}
$$

and

$$
\sigma=(a+b)\left(\left(1+\varepsilon|h|^{2}\right)^{2}|\omega|^{2}-\frac{(1-\varepsilon)^{2}|d h|^{2}}{\left(1+\varepsilon|h|^{2}\right)^{2}}\right) .
$$

ii) Conversely, let $S$ be a Riemann surface, $g: S \longrightarrow \mathbb{S L}(2, \mathbb{C})$ a meromorphic curve and $(h, \omega)$ a pair as above satisfying (18) and such that (20) is a positive definite metric. Then $\psi=g \Omega g^{*}: S \longrightarrow \mathbb{H}^{3}(\Omega$ as in (17)) is a BLW-surface satisfying (31) with induced metric and $\sigma$ given by (19) and (20).

Proof. Let us assume that $\psi(S)$ is not a piece of a horosphere, otherwise the result is easily obtained with $h$ constant. Since $S$ is noncompact and simply connected there exist a global conformal parameter $z$ and, as $\langle d(\psi+\eta), d(\psi+\eta)\rangle$ is a pseudometric on $S$ of constant Gauss curvature $\varepsilon$, then, from the Frobenius theorem, there exists a meromorphic function $h$ on $S$ (holomorphic if $\varepsilon \leq 0$ ) such that

$$
1+\varepsilon|h|^{2}>0 \quad \text { and } \quad\langle d(\psi+\eta), d(\psi+\eta)\rangle=\lambda^{2}|\operatorname{det}(g)|^{2}|d z|^{2}=\frac{4|d h|^{2}}{\left(1+\varepsilon|h|^{2}\right)^{2}},
$$

where we follow the same notation as above.

From this expression, if $h$ has a pole of order $m$, then $d h$ has a pole of order $m+1$ and $\operatorname{det}(g)$ has a zero of order $m-1$. Hence, there exists a meromorphic function $R$ on $S$ (holomorphic if $\varepsilon \leq 0$ ) with

$$
R^{2}=\frac{d h}{\operatorname{det}(g) d z} \neq 0 \quad \text { and } \quad(A R)(B R)_{z}-(B R)(A R)_{z}=h_{z} .
$$

Now, if we replace $A$ and $B$ by $A R$ and $B R$, respectively, in (15) and choose the matrix

$$
g=\left(\begin{array}{cc}
A & \frac{1}{h_{z}} A_{z} \\
B & \frac{1}{h_{z}} B_{z}
\end{array}\right)
$$

with meromorphic coefficients and $\operatorname{det}(g)=1$ on $S$, then the new $\lambda^{2}$ is $4 /\left(1+\varepsilon|h|^{2}\right)^{2}$. Moreover,

$$
g^{-1} d g=\left(\begin{array}{cc}
0 & \frac{1}{h_{z}^{2}}\left(A_{z z} B_{z}-A_{z} B_{z z}\right) \\
h_{z} & 0
\end{array}\right) d z
$$

and we obtain (18) for $\omega=\frac{A_{z z} B_{z}-A_{z} B_{z z}}{h_{z}^{2}} d z$.

From (14) one has that (16) is satisfied for

$$
\Omega=\left(\begin{array}{cc}
\delta \lambda_{z \bar{z}}-\beta \lambda & \delta \lambda_{z} \overline{h_{z}} \\
\delta \lambda_{\bar{z}} h_{z} & \delta \lambda\left|h_{z}\right|^{2}
\end{array}\right) \quad \text { and } \quad \widetilde{\Omega}=\left(\begin{array}{cc}
(1+\beta) \lambda-\delta \lambda_{z \bar{z}} & -\delta \lambda_{z} \overline{h_{z}} \\
-\delta \lambda_{\bar{z}} h_{z} & -\delta \lambda\left|h_{z}\right|^{2}
\end{array}\right)
$$

where $2=\langle\eta, \eta\rangle-\langle\psi, \psi\rangle=\delta \lambda^{2}\left|h_{z}\right|^{2}$. Therefore, (16) and (17) are satisfied. 
Besides,

$$
\begin{aligned}
& d \psi=d\left(g \Omega g^{*}\right)=g\left(\left(\begin{array}{cc}
0 & \omega \\
d h & 0
\end{array}\right) \Omega+d \Omega+\Omega\left(\begin{array}{cc}
0 & d \bar{h} \\
\bar{\omega} & 0
\end{array}\right)\right) g^{*} \\
& =g\left(\begin{array}{cc}
\star & \left(1+\varepsilon|h|^{2}\right) \omega+\frac{1-\varepsilon}{1+\varepsilon|h|^{2}} d \bar{h} \\
\left(1+\varepsilon|h|^{2}\right) \bar{\omega}+\frac{1-\varepsilon}{1+\varepsilon|h|^{2}} d h & 0
\end{array}\right) g^{*}, \\
& d \eta=d\left(g \widetilde{\Omega} g^{*}\right)=g\left(\left(\begin{array}{cc}
0 & \omega \\
d h & 0
\end{array}\right) \widetilde{\Omega}+d \widetilde{\Omega}+\widetilde{\Omega}\left(\begin{array}{cc}
0 & d \bar{h} \\
\bar{\omega} & 0
\end{array}\right)\right) g^{*} \\
& =g\left(\begin{array}{cc}
\star & -\left(1+\varepsilon|h|^{2}\right) \omega+\frac{1+\varepsilon}{1+\varepsilon|h|^{2}} d \bar{h} \\
-\left(1+\varepsilon|h|^{2}\right) \bar{\omega}+\frac{1+\varepsilon}{1+\varepsilon|h|^{2}} d h & 0
\end{array}\right) g^{*}
\end{aligned}
$$

and

$$
d(\psi+\eta)=g\left(\begin{array}{cc}
\star & \frac{2 d \bar{h}}{1+\varepsilon|h|^{2}} \\
\frac{2 d h}{1+\varepsilon|h|^{2}} & 0
\end{array}\right) g^{*} .
$$

Hence, one calculates the induced metric $I$, 19), and the metric $\sigma=a I+b I I$, (20), from

$$
I=\langle d \psi, d \psi\rangle=-\operatorname{det}(d \psi)
$$

and

$$
I I=\langle d \psi,-d \eta\rangle=\frac{1}{2}(\operatorname{det}(d(\psi+\eta))-\operatorname{det}(d \psi)-\operatorname{det}(d \eta)) .
$$

In particular, $\left(1+\varepsilon|h|^{2}\right)^{2}|\omega|^{2}$ is a real expression on $S$ and consequently $\omega$ must be holomorphic.

Following the same notation as in $[2]$ and [6], the pair $(h, \omega)$ given by the above theorem will be called the Weierstrass data associated with the conformal representation of the BLW-surface $\psi$. However, from (18) it is clear that the pair $(h, g)$ could also be called the Weierstrass data of the immersion.

Remark 4. If $S$ is a noncompact, simply connected surface and $\psi_{1}, \psi_{2}: S \longrightarrow \mathbb{H}^{3}$ are two BLW-surfaces satisfying (3) with the same Weierstrass data $(h, \omega)$, then $\psi_{1}$ and $\psi_{2}$ are the same immersion, up to a rigid motion in $\mathbb{H}^{3}$. Indeed, $\psi_{1}=g_{1} \Omega g_{1}^{*}$ and $\psi_{2}=g_{2} \Omega g_{2}^{*}$, where

$$
g_{1}^{-1} d g_{1}=\left(\begin{array}{cc}
0 & \omega \\
d h & 0
\end{array}\right)=g_{2}^{-1} d g_{2}
$$

for some meromorphic curves $g_{1}, g_{2}: S \longrightarrow \mathbb{S L}(2, \mathbb{C})$.

Thus, one has

$$
d\left(g_{1} g_{2}^{-1}\right)=d g_{1} g_{2}^{-1}+g_{1} d\left(g_{2}^{-1}\right)=g_{1}\left(\begin{array}{cc}
0 & \omega \\
d h & 0
\end{array}\right) g_{2}^{-1}+g_{1}\left(\begin{array}{cc}
0 & -\omega \\
-d h & 0
\end{array}\right) g_{2}^{-1}=0
$$

and there exists a constant matrix $g_{0} \in \mathbb{S L}(2, \mathbb{C})$ such that $g_{1}=g_{0} g_{2}$. Consequently, $\psi_{1}=g_{0} \psi_{2} g_{0}^{*}$ as we wanted to prove.

It is interesting to observe that if $\psi: S \longrightarrow \mathbb{H}^{3}$ is an immersion with $H=1$, then $\varepsilon=1$,

$$
\Omega=\left(\begin{array}{cc}
0 & -i \\
-i & i h
\end{array}\right)\left(\begin{array}{cc}
0 & i \\
i & -i \bar{h}
\end{array}\right)
$$


and $\psi=g \Omega g^{*}=F F^{*}$, where

$$
F=g\left(\begin{array}{cc}
0 & -i \\
-i & i h
\end{array}\right) \in \mathbb{S L}(2, \mathbb{C})
$$

that is, the conformal representation becomes the Bryant's representation (see [2], [25]).

Moreover, if the immersion does not lie in a horosphere and we denote by $G$ its hyperbolic Gauss map, then taking $A=G$ and $B=1$ in (15) one gets from (21) and (22)

$$
g=\left(\begin{array}{cc}
i G \sqrt{\frac{d h}{d G}} & \frac{i}{d h} d\left(G \sqrt{\frac{d h}{d G}}\right) \\
i \sqrt{\frac{d h}{d G}} & \frac{i}{d h} d\left(\sqrt{\frac{d h}{d G}}\right)
\end{array}\right) .
$$

Thus, by considering the new parameter $\zeta=h$ where that is possible, one has

$$
F=\frac{1}{2 G_{\zeta}^{3 / 2}}\left(\begin{array}{cc}
2 G_{\zeta}^{2}-G_{G_{\zeta \zeta}} & 2 G_{\zeta}\left(G-\zeta G_{\zeta}\right)+\zeta G G_{\zeta \zeta} \\
-G_{\zeta \zeta} & 2 G_{\zeta}+\zeta G_{\zeta \zeta}
\end{array}\right)
$$

that is, we recover Small's formula for surfaces with constant mean curvature one (see [10], 23]).

\section{COMPleteness OF THE IMMERsions}

From Remark 3 it is clear that the Gauss curvature $K_{I}$ of a BLW-surface $\psi$ : $S \longrightarrow \mathbb{H}^{3}$ is nonnegative on $S$ or nonpositive everywhere.

Moreover, if $S$ is noncompact, then, from (16), (17), (19) and (20), its Gaussian curvature $K_{I}=K-1$ can be calculated as

$$
K_{I}=\frac{-4 \varepsilon|d h|^{2}}{\left(1+\varepsilon|h|^{2}\right)^{4}|\omega|^{2}-(1-\varepsilon)^{2}|d h|^{2}}
$$

and its mean curvature is given by

$$
H=1+\frac{2(1-\varepsilon)|d h|^{2}}{\left(1+\varepsilon|h|^{2}\right)^{4}|\omega|^{2}-(1-\varepsilon)^{2}|d h|^{2}} .
$$

In particular, a point $p \in S$ is umbilical if and only if $d h(p)=0$ or $\omega(p)=0$.

We shall study separately the cases of complete immersions with nonpositive and nonnegative Gauss curvature.

3.1. Completeness with nonnegative Gauss curvature. First, we give some examples of complete BLW-surfaces and then we classify them as the only nontotally umbilical complete BLW-surfaces with nonnegative Gauss curvature.

Let us consider $S=\mathbb{C}, h(z)=z+c_{0}$ and $\omega(z)=c_{1} d z$ for some complex constants $c_{0}$ and $c_{1}$ :

1. If we take $a=0$ and $b=1$, then, from Theorem 2, one obtains the flat immersion

$$
\psi(u, v)=\left(\begin{array}{cc}
\frac{1+r^{2}}{2 r} e^{-u} & \frac{1-r^{2}}{2 r} e^{-i v} \\
\frac{1-r^{2}}{2 r} e^{i v} & \frac{1+r^{2}}{2 r} e^{u}
\end{array}\right),
$$

with $r=\sqrt{\left|c_{1}\right|}, u+i v=2 \sqrt{c_{1}} z$ and $\sigma=\left(\left|c_{1}\right|^{2}-1\right)|d z|^{2}$ positive definite when $\left|c_{1}\right|>1$. Moreover, the induced metric $I=c_{1} d z^{2}+\left(1+\left|c_{1}\right|^{2}\right)|d z|^{2}+$ $\overline{c_{1}} d \bar{z}^{2} \geq\left(\left|c_{1}\right|-1\right)^{2}|d z|^{2}$ is complete. 
2. If we put $a=0$ and $b=-1$, then (24) is a complete flat immersion with $0<\left|c_{1}\right|<1$.

In any case, the immersion given by (24) may be regarded as the set of points at a fixed distance from the geodesic

$$
\gamma(t)=\left(\begin{array}{cc}
e^{-t} & 0 \\
0 & e^{t}
\end{array}\right)
$$

namely, a hyperbolic cylinder.

Theorem 3. Let $\psi: S \longrightarrow \mathbb{H}^{3}$ be a complete BLW-surface with nonnegative Gauss curvature $K_{I}$. Then $\psi(S)$ is a totally umbilical round sphere, a horosphere or a hyperbolic cylinder.

Proof. We can assume that $S$ is simply connected, otherwise we would pass to the universal covering surface of $S$. Moreover, if $S$ is compact, then, from Remark 2 $\psi(S)$ is a totally umbilical round sphere.

First, let us suppose that $\psi$ is a nonflat immersion, that is, there exists a point on $S$ such that $K_{I}>0$ (in particular, from Remark $3 a<0$ ).

If $a+b=-1$, then from (3) and since $H^{2} \geq K$, we have that $K_{I} \geq-4 a / b^{2}>0$. Therefore, from Bonnet's theorem, $S$ must be compact.

If $a+b=1, \varepsilon<0$ and from (19)

$$
\frac{1}{2} I \leq \tau=\frac{(1-\varepsilon)^{2}|d h|^{2}}{\left(1+\varepsilon|h|^{2}\right)^{2}}+\left(1+\varepsilon|h|^{2}\right)^{2}|\omega|^{2}
$$

and $\tau$ must be a complete metric on $S$. Besides, using that $\sigma$ is a positive definite metric and that the bounded holomorphic function $h$ satisfies that $1+\varepsilon|h|^{2}<1$, we obtain $\tau \leq 2\left(1+\varepsilon|h|^{2}\right)^{2}|\omega|^{2} \leq 2|\omega|^{2}$. Therefore, $2|\omega|^{2}$ is a flat complete metric conformal to $\sigma$. Thus, $S$ is conformally equivalent to the complex plane $\mathbb{C}$ and $h$ must be constant, that is, $\psi(S)$ is a horosphere.

On the other hand, if $\psi$ is a flat immersion, then $a=0=\varepsilon$ and from (19)

$$
\tau=|d h|^{2}+|\omega|^{2}
$$

is a complete metric. Since $\sigma$ is a positive definite metric one has that if $b=1$ (respectively, $b=-1$ ), then $\tau \leq 2|\omega|^{2}$ (resp. $\tau \leq 2|d h|^{2}$ ) and, hence, there exists a complete conformal flat metric on $S$. Thus, $S$ is conformally equivalent to $\mathbb{C}$ and the function $d h / \omega$ (or $\omega / d h)$ is constant with modulus less than one when $b=1$ (or $b=-1$ ).

Therefore, $\psi(S)$ is a horosphere or $\omega=c_{1} d h$ for a complex constant $c_{1} \neq 0$ with $\left|c_{1}\right| \neq 1$. Thus, in the second case $d h \neq 0$ everywhere because $\sigma$ is nondegenerate and we can take the new conformal parameter $\zeta$ such that $d \zeta=d h$. Then one obtains $h(\zeta)=\zeta+c_{0}$ and $\omega(\zeta)=c_{1} d \zeta$ for a constant $c_{0} \in \mathbb{C}$ and $\psi$ lies on a hyperbolic cylinder.

3.2. Completeness with nonpositive Gauss curvature. A Plateau problem at infinity. Now, we focus our attention on the study of the completeness of the immersions with nonpositive Gauss curvature. We remark that given a simply connected surface $S$ and a nonflat BLW-surface $\psi: S \longrightarrow \mathbb{H}^{3}$ with nonpositive Gauss curvature, then $\mathrm{S}$ cannot be compact and $a>0$. Therefore, the immersion lies in the set $R_{3}$ or $R_{4}$.

In the last case, the geometry of the surface is similar to the one of a surface with constant mean curvature $H=1$. In fact, if $(h, \omega)$ are the Weierstrass data 
for a BLW-surface $\psi_{0}$ in $R_{4}$ then $\varepsilon>0$ and $\left(1+\varepsilon|h|^{2}\right)|\omega|^{2}$ is a positive definite metric because $\sigma$ is also. Moreover, we obtain a new associated immersion $\psi_{1}$ with constant mean curvature one and Weierstrass data $(\sqrt{\varepsilon} h, \omega)$. If $\psi_{0}$ is a complete immersion, then, from (19) and (20), $\left(1+\varepsilon|h|^{2}\right)|\omega|^{2}$ is also a complete metric, that is, $\psi_{1}$ is a complete immersion. Thus, the study of complete BLW-surfaces in $R_{4}$ can be reduced to the study of complete immersions with constant mean curvature one. Many things are known in this case and some very interesting results were proved in [2], 3] and [25].

However, the geometry of the surface is very different when the immersion lies in $R_{3}$. For instance,

Lemma 3. Let $\psi: S \longrightarrow \mathbb{H}^{3}$ be a complete BLW-surface in $R_{3}$ with Weierstrass data $(h, \omega)$. Then, $S$ is conformally equivalent to the unit disk $\mathbb{D}$ and $h$ is a global diffeomorphism onto $\mathbb{D}_{\varepsilon}=\left\{z \in \mathbb{C}:|z|^{2}<-1 / \varepsilon\right\}$.

Proof. Since the induced metric is complete and $\sigma$ is positive definite, from (19) and (20)

$$
\frac{1}{2} I \leq \frac{(1-\varepsilon)^{2}|d h|^{2}}{\left(1+\varepsilon|h|^{2}\right)^{2}}+\left(1+\varepsilon|h|^{2}\right)^{2}|\omega|^{2} \leq 2 \frac{(1-\varepsilon)^{2}|d h|^{2}}{\left(1+\varepsilon|h|^{2}\right)^{2}}
$$

and the metric $4|d h|^{2} /\left(1+\varepsilon|h|^{2}\right)^{2}$ must be complete. Therefore, $h: S \longrightarrow \mathbb{D}_{\varepsilon}$ is bijective and $S$ is conformally equivalent to the unit disk.

From the above lemma, given a complete BLW-surface $\psi: S \longrightarrow \mathbb{H}^{3}$ with Weierstrass data $(h, \omega)$ we can consider, up to a change of parameter, $S=\mathbb{D}$ and $h(z)=z / \sqrt{-\varepsilon}$.

Moreover, from (20), $\sigma$ is positive definite if and only if

$$
|\omega|<\frac{1-\varepsilon}{\sqrt{-\varepsilon}} \frac{|d z|}{\left(1-|z|^{2}\right)^{2}}, \quad z \in \mathbb{D} .
$$

On the other hand, if we consider the hyperbolic Gauss map of the immersion $G$ and take $A=G, B=1$ in (15), one gets from (21) and (22)

$$
g=\left(\begin{array}{cc}
i G \sqrt{\frac{d h}{d G}} & \frac{i}{d h} d\left(G \sqrt{\frac{d h}{d G}}\right) \\
i \sqrt{\frac{d h}{d G}} & \frac{i}{d h} d\left(\sqrt{\frac{d h}{d G}}\right)
\end{array}\right)=\left(\begin{array}{cc}
\frac{i G}{\sqrt[4]{-\varepsilon} \sqrt{G_{z}}} & i \sqrt[4]{-\varepsilon}\left(\frac{G}{\sqrt{G_{z}}}\right)_{z} \\
\frac{i}{\sqrt[4]{-\varepsilon} \sqrt{G_{z}}} & i \sqrt[4]{-\varepsilon}\left(\frac{1}{\sqrt{G_{z}}}\right)_{z}
\end{array}\right) .
$$

Thus,

$$
g^{-1} d g=\left(\begin{array}{cc}
0 & \sqrt{-\varepsilon} \frac{3 G_{z z}^{2}-2 G_{z} G_{z z z}}{4 G_{z}^{2}} \\
\frac{1}{\sqrt{-\varepsilon}} & 0
\end{array}\right) d z
$$

and from (18) and (25), the Schwarzian derivative of $G$ satisfies

$$
\{G, z\}:=\frac{d}{d z}\left(\frac{G_{z z}}{G_{z}}\right)-\frac{1}{2}\left(\frac{G_{z z}}{G_{z}}\right)^{2}=\frac{2 G_{z} G_{z z z}-3 G_{z z}^{2}}{2 G_{z}^{2}}=\frac{-2}{\sqrt{-\varepsilon}} \frac{\omega}{d z}
$$

and

$$
|\{G, z\}|<\frac{2(1-\varepsilon)}{-\varepsilon} \frac{1}{\left(1-|z|^{2}\right)^{2}}, \quad z \in \mathbb{D} .
$$


Consequently, $G$ is a local diffeomorphism with bounded Schwarzian derivative. Otherwise, there would exist a point $z_{0}$ such that $G$ is not locally one-to-one and we could write $G_{z}$ in a neighbourhood of $z_{0}$ as

$$
G_{z}=\left(z-z_{0}\right)^{k} \sum_{n=0}^{\infty} c_{n}\left(z-z_{0}\right)^{n}
$$

with $c_{0} \neq 0$ and an integer $k \neq 0$. Hence

$$
\{G, z\}=-\frac{k(k+2)}{2} \frac{1}{\left(z-z_{0}\right)^{2}}-\frac{k c_{1}}{2 c_{0}} \frac{1}{z-z_{0}}+\widetilde{h}(z),
$$

where $\widetilde{h}$ is a holomorphic function in a neighbourhood of $z_{0}$. But, either $\{G, z\}=\infty$ at $z_{0}$ which contradicts the above inequality or $k=-2$ and $G$ has a pole of order one at $z_{0}$, that is, $G$ is locally one to one.

The above considerations are summarized in the following result:

Theorem 4. Let $S$ be a simply connected surface and let $\psi: S \longrightarrow \mathbb{H}^{3}$ be a complete $B L W$-surface in $R_{3}$. Then one has

(i) $S$ can be identified with $\mathbb{D}$ and its Weierstrass data $h$ can be taken as $h(z)=$ $z / \sqrt{-\varepsilon}$

(ii) the hyperbolic Gauss map $G: \mathbb{D} \longrightarrow \mathbb{C} \cup\{\infty\}$ is a local diffeomorphism satisfying

$$
|\{G, z\}|<\frac{2(1-\varepsilon)}{-\varepsilon} \frac{1}{\left(1-|z|^{2}\right)^{2}}, \quad z \in \mathbb{D},
$$

(iii) the immersion can be recovered as

$$
\begin{aligned}
& \frac{1}{\sqrt{-\varepsilon}\left|G_{z}\right|\left(1-|z|^{2}\right)} \\
& \quad \times\left(\begin{array}{cc}
|G|^{2}-\varepsilon\left|-G \mathcal{A}+G_{z}\left(1-|z|^{2}\right)\right|^{2} & \left(1-\varepsilon|\mathcal{A}|^{2}\right) G+\varepsilon G_{z}\left(1-|z|^{2}\right) \overline{\mathcal{A}} \\
\left(1-\varepsilon|\mathcal{A}|^{2}\right) \bar{G}+\varepsilon \overline{G_{z}}\left(1-|z|^{2}\right) \mathcal{A} & 1-\varepsilon|\mathcal{A}|^{2}
\end{array}\right)
\end{aligned}
$$

and its Gauss map $\eta$ as

$$
\begin{aligned}
& \frac{1}{\sqrt{-\varepsilon}\left|G_{z}\right|\left(1-|z|^{2}\right)} \\
& \times\left(\begin{array}{cc}
|G|^{2}+\varepsilon\left|-G \mathcal{A}+G_{z}\left(1-|z|^{2}\right)\right|^{2} & \left(1+\varepsilon|\mathcal{A}|^{2}\right) G-\varepsilon G_{z}\left(1-|z|^{2}\right) \overline{\mathcal{A}} \\
\left(1+\varepsilon|\mathcal{A}|^{2}\right) \bar{G}-\varepsilon \overline{G_{z}}\left(1-|z|^{2}\right) \mathcal{A} & 1+\varepsilon|\mathcal{A}|^{2}
\end{array}\right) \\
& \text { with } \mathcal{A}=\frac{G_{z z}}{2 G_{z}}\left(1-|z|^{2}\right)-\bar{z}
\end{aligned}
$$

Conversely, let $G: \mathbb{D} \longrightarrow \mathbb{C} \cup\{\infty\}$ be a meromorphic map. Then, if $G$ satisfies (27) one has that (28) is a BLW-surface in $R_{3}$ with hyperbolic Gauss map $G$ and Weierstrass data $\left(z / \sqrt{-\varepsilon},-\frac{1}{2} \sqrt{-\varepsilon}\{G, z\} d z\right)$. Moreover, if

$$
|\{G, z\}| \leq \frac{b_{0}}{\left(1-|z|^{2}\right)^{2}}, \quad z \in \mathbb{D},
$$

with $b_{0}<\frac{2(1-\varepsilon)}{-\varepsilon}$, then the immersion is complete. 
Proof. Let $\psi: S \longrightarrow \mathbb{H}^{3}$ be a complete BLW-surface in $R_{3}$ and $S$ simply connected. Then, we can assume that (i) and (ii) are satisfied and, following Theorem 2 , the immersion and its Gauss map can be recovered as in (16) and (17), where $g$ is given as in (22) for some meromorphic functions $A$ and $B$.

Thus,

$$
\psi=\frac{1}{1-|z|^{2}}\left(\begin{array}{cc}
|A|^{2}-\varepsilon \mathcal{A}_{1} \overline{\mathcal{A}_{1}} & A \bar{B}-\varepsilon \mathcal{A}_{1} \overline{\mathcal{A}_{2}} \\
\bar{A} B-\varepsilon \overline{\mathcal{A}_{1}} \mathcal{A}_{2} & |B|^{2}-\varepsilon \mathcal{A}_{2} \overline{\mathcal{A}_{2}}
\end{array}\right)
$$

where

$$
\mathcal{A}_{1}=A \bar{z}+A_{z}\left(1-|z|^{2}\right), \quad \mathcal{A}_{2}=B \bar{z}+B_{z}\left(1-|z|^{2}\right) .
$$

Now, using that $G=A / B, g \in \mathbb{S L}(2, \mathbb{C})$, that is, $\sqrt{-\varepsilon}\left(A B_{z}-B A_{z}\right)=1$ and

$$
G_{z}=\frac{-1}{\sqrt{-\varepsilon} B^{2}}, \quad \frac{G_{z z}}{G_{z}}=-2 \frac{B_{z}}{B},
$$

one has

$$
\begin{aligned}
& \mathcal{A}_{2}=B\left(\bar{z}+\frac{B_{z}}{B}\left(1-|z|^{2}\right)\right)=-B \mathcal{A}, \\
& \mathcal{A}_{1}=G \mathcal{A}_{2}-\frac{1}{\sqrt{-\varepsilon} B}\left(1-|z|^{2}\right)=B\left(-G \mathcal{A}+G_{z}\left(1-|z|^{2}\right)\right),
\end{aligned}
$$

and bearing in mind that $\sqrt{-\varepsilon}|B|^{2}\left|G_{z}\right|=1$ from (31), one obtains (28). Analogously, one can compute (29).

From Theorem 2, the converse part is a straightforward computation taking $g$ as in (26). Moreover, if $G$ satisfies (30), the induced metric $I$ can be estimated as follows:

$$
\begin{aligned}
I & =\left|\frac{(1-\varepsilon) d h}{1+\varepsilon|h|^{2}}+\left(1+\varepsilon|h|^{2}\right) \bar{\omega}\right|^{2} \geq\left(\frac{(1-\varepsilon)|d h|}{1+\varepsilon|h|^{2}}-\left(1+\varepsilon|h|^{2}\right)|\omega|\right)^{2} \\
& =\left(\frac{1-\varepsilon}{\sqrt{-\varepsilon}\left(1-|z|^{2}\right)}-\left(1-|z|^{2}\right) \frac{\sqrt{-\varepsilon}}{2}|\{G, z\}|\right)^{2}|d z|^{2} \\
& \geq\left(\frac{1-\varepsilon}{\sqrt{-\varepsilon}}-\frac{\sqrt{-\varepsilon}}{2} b_{0}\right)^{2} \frac{|d z|^{2}}{\left(1-|z|^{2}\right)^{2}} .
\end{aligned}
$$

Since $b_{0}<\frac{2(1-\varepsilon)}{-\varepsilon}$, then $\frac{1-\varepsilon}{\sqrt{-\varepsilon}}-\frac{\sqrt{-\varepsilon}}{2} b_{0}$ is a positive number and the induced metric is, up to a constant, greater than or equal to the Poincaré metric on the unit disk. Hence, the immersion is complete.

Remark 5. Let $\psi_{0}: \mathbb{D} \longrightarrow \mathbb{H}^{3}$ be a BLW-surface in $R_{3}$ with Weierstrass data $h_{0}(z)=z / \sqrt{-\varepsilon}$ and hyperbolic Gauss map $G_{0}$. If we consider the new immersion $\psi_{1}: \mathbb{D} \longrightarrow \mathbb{H}^{3}$ with $h_{1}(\zeta)=\zeta / \sqrt{-\varepsilon}$ and $G_{1}(\zeta)=G_{0}(\varphi(\zeta))$, where $\varphi: \mathbb{D} \longrightarrow \mathbb{D}$ is a conformal equivalence, then $\psi_{0}(\varphi(\zeta))=\psi_{1}(\zeta)$.

Indeed, since $\varphi$ is a biholomorphic map onto the unit disk

$$
\varphi(\zeta)=e^{i \theta} \frac{\zeta+\zeta_{0}}{\overline{\zeta_{0}} \zeta+1}, \quad \zeta \in \mathbb{D}
$$

for some $\zeta_{0} \in \mathbb{D}, \theta \in \mathbb{R}$ and

$$
\frac{\varphi_{\zeta}(\zeta)}{1-|\varphi(\zeta)|^{2}}=e^{i \theta} \frac{1+\zeta_{0} \bar{\zeta}}{1+\bar{\zeta}_{0} \zeta} \frac{1}{1-|\zeta|^{2}} .
$$




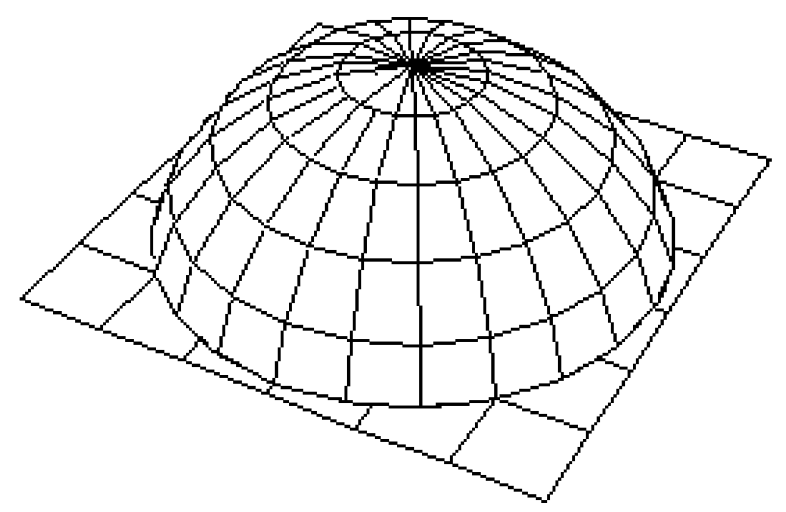

FIgURE 2. Totally umbilical BLW-surfaces.

Thus, one has

$$
\begin{aligned}
G_{1 \zeta}(\zeta)\left(1-|\zeta|^{2}\right) & =e^{i \theta} \frac{1+\zeta_{0} \bar{\zeta}}{1+\overline{\zeta_{0} \zeta}} G_{0 z}(\varphi(\zeta))\left(1-|\varphi(\zeta)|^{2}\right) \\
\mathcal{A}_{\psi_{1}}(\zeta) & =e^{i \theta} \frac{1+\zeta_{0} \bar{\zeta}}{1+\overline{\zeta_{0} \zeta}} \mathcal{A}_{\psi_{0}}(\varphi(\zeta))
\end{aligned}
$$

and the above equality is clear from (28).

Now, for the representation of some surfaces and the study of its geometric behaviour, we will identify the hyperbolic space $\mathbb{H}^{3} \subset \mathbb{L}^{4}$ with the upper half-space of $\mathbb{R}^{3}$ given by $\left\{\left(x_{1}, x_{2}, x_{3}\right) \in \mathbb{R}^{3}: x_{3}>0\right\}$ by considering the map

$$
\left(x_{0}, x_{1}, x_{2}, x_{3}\right) \longrightarrow \frac{1}{x_{0}+x_{3}}\left(x_{1}, x_{2}, 1\right) .
$$

Under this identification the hyperbolic metric is given by

$$
d s^{2}=\frac{1}{x_{3}^{2}}\left(d x_{1}^{2}+d x_{2}^{2}+d x_{3}^{2}\right)
$$

and its ideal boundary $\mathbb{S}_{\infty}^{2}$ is identified with the one point compactification of the plane $\Pi \equiv\left\{x_{3}=0\right\}$, that is, $\mathbb{S}_{\infty}^{2}=\Pi \cup\{\infty\}$. Thus, the asymptotic boundary of a set $\Sigma \subset \mathbb{H}^{3}$ is

$$
\partial_{\infty}(\Sigma)=\operatorname{cl}(\Sigma) \cap \mathbb{S}_{\infty}^{2}
$$

where $\operatorname{cl}(\Sigma)$ is the closure of $\Sigma$ in $\left\{x_{3} \geq 0\right\} \cup\{\infty\}$, that is, in the one point compactification of $\mathbb{H}^{3} \cup \Pi$.

Example 1. Let $\psi: \mathbb{D} \longrightarrow \mathbb{H}^{3}$ be a complete totally umbilical BLW-surface with $\varepsilon<0$. From the comments at the beginning of Section $\S 3$ and from Lemma 3, we have that the Weierstrass data of the immersion are $(z / \sqrt{-\varepsilon}, 0), z \in \mathbb{D}$. In that case $G(z)=r z, r>0$, is a holomorphic solution of $\{G, z\}=0$. Consequently, from Theorem 4 and (32) the immersion is isometric to the following one:

$$
\psi_{1}(u, v)=\frac{r}{1-\varepsilon\left(u^{2}+v^{2}\right)}\left\{(1-\varepsilon) u,(1-\varepsilon) v, \sqrt{-\varepsilon}\left(1-u^{2}-v^{2}\right)\right\},
$$

$z=u+i v \in \mathbb{D}$. It is clear that the asymptotic boundary of $\psi_{1}$ is a circle in $\Pi \cup\{\infty\}$ of radius $r$; see Figure 2. 


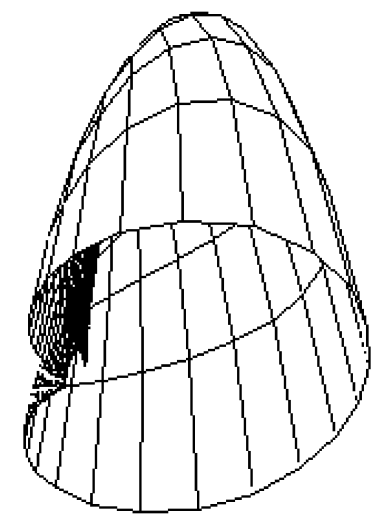

FIGURE 3. BLW-surface with nonregular asymptotic boundary.

Example 2. For $\varepsilon=-1 / 4$, let $\psi: \mathbb{D} \longrightarrow \mathbb{H}^{3}$ be the BLW-surface associated with the Weierstrass data $\left(2 z, 3 d z /\left(8\left(1-z^{2}\right)\right)\right), z \in \mathbb{D}$. Since a solution of the equation $2\{G, z\}=-3 /\left(1-z^{2}\right)$ is the holomorphic map $G(z)=(1-z)^{2} / 4, z \in \mathbb{D}$, we conclude from Theorem 4 and (32) that the immersion $\psi$ is regular.

Consider the new conformal parameter $x+i y=\varphi(z)=2 i /(1-z)$ and take $\tau$ the isometry of $\mathbb{H}^{3}$ given by the Euclidean inversion with respect to the unit sphere centered at $(0,0,0)$. Then $\tau \circ \psi$ is parametrized as $\psi_{2} \circ \varphi$, where

$$
\begin{aligned}
\psi_{2}(x, y)= & \left\{\frac{1-4 y+8 y^{3}+8 x^{2}-5 x^{4}+y^{2}\left(5-3 x^{2}\right)}{D},\right. \\
& \left.\frac{-2 x\left(6 y^{2}+2 y^{3}+2 x^{2}+3 y\left(-1+x^{2}\right)\right)}{D}, \frac{8(-1+y)\left(y^{2}+x^{2}\right)^{\frac{3}{2}}}{D}\right\},
\end{aligned}
$$

and $D:=1-12 y^{5}+8 y^{6}+11 x^{2}-x^{4}+5 x^{6}+y^{3}\left(16-24 x^{2}\right)+3 y^{4}\left(3+7 x^{2}\right)+$ $2\left(y+3 y x^{2}\right)^{2}-4 y\left(1+3 x^{4}\right)$.

It is clear (see Figure 3) that the immersion $\tau \circ \psi$ is regular, but its asymptotic boundary is a nonregular Jordan curve.

Example 3. Let $\varepsilon=-1 /\left(4 n^{2}+4 n\right)$, with $n$ a positive integer and let $\psi: \mathbb{D} \longrightarrow \mathbb{H}^{3}$ be the BLW-surface associated with the Weierstrass data

$$
\left(z / \sqrt{-\varepsilon},-\frac{n+1}{2 n} \sqrt{-\varepsilon} d z /\left(1-z^{2}\right)^{2}\right), \quad z \in \mathbb{D} .
$$

Since a solution of the equation $n\{G, z\}=-(n+1) /\left(1-z^{2}\right)^{2}$ is the meromorphic map

$$
G(z)=\tan \left(\frac{(1+2 n)\left(-\pi+2 i \log \left(\frac{1+z}{1-z}\right)\right)}{4}\right),
$$

$z \in \mathbb{D}$, it is not difficult to see that the immersion is not embedded and has as asymptotic boundary the set $\left\{\left(0, x_{2}, 0\right): x_{2} \in \mathbb{R}\right\} \cup\{\infty\}$ in $\Pi \cup\{\infty\}$.

In fact, for $n=1$ if we consider $\tau$ the isometry of $\mathbb{H}^{3}$ given by the Euclidean inversion with respect to the unit sphere centered at $(1,1,0)$, then the asymptotic boundary of $\tau \circ \psi$ is the circle of radius $1 / 2$ centered at $1 / 2+i$ on $\Pi \cup\{\infty\}$. Moreover, 


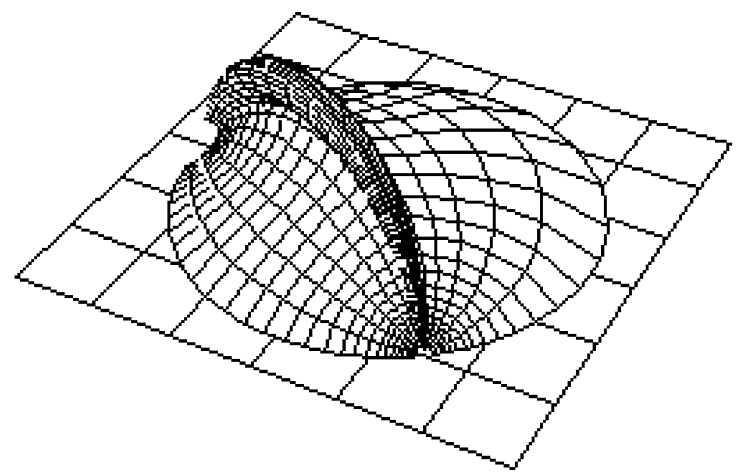

Figure 4. Nonembedded BLW-surface with embedded asymptotic boundary.

by taking the new conformal parameter $x+i y=\varphi(z)=\log \left(\frac{1+z}{1-z}\right)$, the immersion $\tau \circ \psi$ is parametrized as $\psi_{3} \circ \varphi$, where

$$
\begin{aligned}
\psi_{3}(x, y)= & \frac{1}{B}\{4 \cos (y)-4 \cos (3 y)+\cos (5 y) \\
& +2(13+4 \cos (2 y))(\cosh (3 x)-\sinh (3 x)), \\
2 & (4 \cos (y)-4 \cos (3 y)+\cos (5 y)) \\
& +(13+4 \cos (2 y))(2 \cosh (3 x)-\sinh (3 x)), 12 \sqrt{2} \cos (y)\}
\end{aligned}
$$

and $B=8 \cos (y)-8 \cos (3 y)+2 \cos (5 y)+3(13+4 \cos (2 y)) \cosh (3 x)-4 \sin (y)+$ $4 \sin (3 y)-\sin (5 y)-2(13+4 \cos (2 y)) \sinh (3 x)$; see Figure 4.

Example 4. We fix $\varepsilon=-1 / 8$ and take the BLW-surface $\psi: \mathbb{D} \longrightarrow \mathbb{H}^{3}$ associated with the Weierstrass data $\left(2 \sqrt{2} z,-5 /\left(2 \sqrt{2}\left(1-z^{2}\right)^{2}\right) d z\right), z \in \mathbb{D}$. Since a solution of the equation $\{G, z\}=10 /\left(1-z^{2}\right)^{2}$ is the holomorphic map

$$
G(z)=\tan \left(\log \left(\frac{z+1}{z-1}\right)\right),
$$

$z \in \mathbb{D}$, from Theorem 4 and (32) $\psi$ is a regular immersion and its asymptotic boundary are two disjoint circles in $\Pi \cup\{\infty\}$.

By taking the new conformal parameter $x+i y=\varphi(z)=\log \left(\frac{z+1}{z-1}\right)$, the immersion $\psi$ is parametrized as $\psi_{4} \circ \varphi$, where

$$
\begin{aligned}
\psi_{4}(x, y) & =\left\{\frac{(-13+5 \cos (2 y)) \sin (2 x)}{\cos (2 x)(-13+5 \cos (2 y))-3(7+\cos (2 y)) \cosh (2 y)-4 \sin (2 y) \sinh (2 y)},\right. \\
& \frac{4 \cosh (2 y) \sin (2 y)+3(7+\cos (2 y)) \sinh (2 y)}{\cos (2 x)(13-5 \cos (2 y))+3(7+\cos (2 y)) \cosh (2 y)+4 \sin (2 y) \sinh (2 y)}, \\
& \left.\frac{16 \sqrt{2} \cos (y)}{\cos (2 x)(-13+5 \cos (2 y))-3(7+\cos (2 y)) \cosh (2 y)-4 \sin (2 y) \sinh (2 y)}\right\} .
\end{aligned}
$$




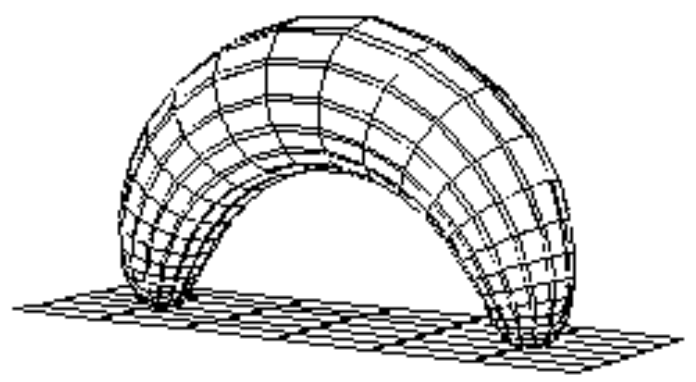

FigURE 5. BLW-surface with a nonconnected embedded asymptotic boundary.

It is clear that the immersion can be considered as an embedding of a cylinder with two disjoint circles in $\Pi \cup\{\infty\}$ as its asymptotic boundary; see Figure 5 .

For the study of these surfaces at infinity we pose the following Plateau problem:

Given $\varepsilon_{0}<0$ and a Jordan curve $\Gamma$ on $\mathbb{S}_{\infty}^{2} \equiv \Pi \cup\{\infty\}$, find a complete BLW-surface $\psi: S \longrightarrow \mathbb{H}^{3}$ satisfying

$$
2\left(-\varepsilon_{0}\right)(H-1)+\left(\varepsilon_{0}-1\right) K_{I}=0
$$

with $\Gamma$ as its asymptotic boundary.

Since every preserving orientation isometry of $\mathbb{H}^{3}$ induces a Möbius transformation on $\mathbb{S}_{\infty}^{2}$ (see, for instance, [24]), then we will assume that, up to a Möbius transformation, the Jordan curve $\Gamma$ lies on $\Pi$.

Proposition 1. Let $\Gamma$ be a Jordan curve on $\Pi \equiv\left\{x_{3}=0\right\} \subset \mathbb{R}^{3}$, int $(\Gamma)$ the bounded component of $\Pi \backslash \Gamma$ and $G: \mathbb{D} \longrightarrow \mathbb{I}_{\Gamma}$ a conformal equivalence, where $\mathbb{I}_{\Gamma}=\left\{x_{1}+i x_{2} \in \mathbb{C}:\left(x_{1}, x_{2}, 0\right) \in \operatorname{int}(\Gamma)\right\}$. Then, if $G$ satisfies (27) for a fixed $\varepsilon<0$, the immersion $\psi: \mathbb{D} \longrightarrow \mathbb{H}^{3}$ associated with $G$ by Theorem 4 lies in the region $R_{3}$ and it is a solution to the Plateau problem for the Jordan curve $\Gamma$.

Moreover,

1. $\psi$ has a continuous extension $\widetilde{\psi}$ to the closure $\overline{\mathbb{D}}$ of $\mathbb{D}$, such that $\widetilde{\psi}_{\mid \partial \overline{\mathbb{D}}}$ : $\partial \overline{\mathbb{D}} \longrightarrow \Gamma$ is a homeomorphism. If $\Gamma$ is a $C^{\infty}$ smooth Jordan curve, then $\psi$ and its derivatives have continuous extensions to $\overline{\mathbb{D}}$,

2. if $\psi$ is an embedding and $\Gamma$ is a differentiable curve such that each tangent line to $\Gamma$ does not pass through a fixed point $p \in \operatorname{int}(\Gamma)$, then the bounded domain by $\widetilde{\psi}(\overline{\mathbb{D}}) \cup \operatorname{int}(\Gamma)$, $\mathcal{O}$, is starshaped from $p$ in $\mathbb{R}^{3}$.

Proof. First, we observe that $G$ has an extension to a homeomorphism $\widetilde{G}$ of the closed disk $\overline{\mathbb{D}}$ onto the closure of $\mathbb{I}_{\Gamma}[8$ Theorem II.3.4]. Moreover, if $\Gamma$ is differentiable, the above extension is a diffeomorphism [8, Theorem II.3.5].

On the other hand, from 28) and (32), the immersion can be written as

$$
\psi(z)=\left(G+\varepsilon G_{z}\left(1-|z|^{2}\right) \frac{\overline{\mathcal{A}}}{1-\varepsilon|\mathcal{A}|^{2}}, \sqrt{-\varepsilon} \frac{\left|G_{z}\right|\left(1-|z|^{2}\right)}{1-\varepsilon|\mathcal{A}|^{2}}\right) .
$$


Thus, if we consider the extension $\widetilde{\psi}$ of $\psi$ in $\mathbb{R}^{3}$ such that $\widetilde{\psi}(z)=(\widetilde{G}(z), 0)$ when $|z|=1, \widetilde{\psi}$ is a continuous map on $\overline{\mathbb{D}}$ since

$$
1-\varepsilon|\mathcal{A}|^{2} \geq 1, \quad \frac{|\mathcal{A}|}{1-\varepsilon|\mathcal{A}|^{2}} \text { is bounded }
$$

and $\left|G_{z}\right|\left(1-|z|^{2}\right) \rightarrow 0$ when $|z| \rightarrow 1$ (see 4, Lemma 14.2.8]). In particular, $\psi$ is a solution to the Plateau problem for the Jordan curve $\Gamma$.

Moreover, if $\Gamma$ is differentiable, then $\widetilde{G}$ is also differentiable and $\widetilde{G}_{z} \neq 0$ for $|z|=1$, so $\mathcal{A}$ is well defined on $\overline{\mathbb{D}}$ and $\widetilde{\psi}$ is a smooth extension of $\psi$ in $\mathbb{R}^{3}$.

Now, let us assume that $\psi$ is an embedding and that $\Gamma$ is a differentiable curve such that each tangent line to $\Gamma$ does not pass through a fixed point $p \in \operatorname{int}(\Gamma)$. We consider

$B_{t}^{-}=\left\{q \in \mathbb{R}^{3}:\|q-p\| \leq t\right\} \cap\left\{x_{3} \geq 0\right\}, \quad B_{t}^{+}=\left\{q \in \mathbb{R}^{3}:\|q-p\| \geq t\right\} \cap\left\{x_{3} \geq 0\right\}$, where $\|$.$\| denotes the usual Euclidean norm and H_{t}=B_{t}^{-} \cap B_{t}^{+}$the closed Euclidean hemisphere with radius $t$ and centered at $p$.

The Euclidean inversion $I_{t}$ with respect to $H_{t}$ is a hyperbolic reflection when $I_{t}$ is restricted to $\mathbb{H}^{3}$ and so we can use the Alexandrov reflection principle. That is, let

$$
\Sigma_{t}^{-}=\widetilde{\psi}(\overline{\mathbb{D}}) \cap B_{t}^{-}, \quad \Sigma_{t}^{+}=I_{t}\left(\widetilde{\psi}(\overline{\mathbb{D}}) \cap B_{t}^{+}\right) .
$$

Since $\widetilde{\psi}(\overline{\mathbb{D}})$ is compact, there exists $t_{0}$ such that $\widetilde{\psi}(\overline{\mathbb{D}}) \subset B_{t_{0}}^{-}$. Therefore, in a standard way, if $\mathcal{O}$ was not starshaped from $p$, then, when the radius decreases from $t_{0}$ to 0 , there would exist a first time $t_{1}$ such that either $\Sigma_{t_{1}}^{-}$and $\Sigma_{t_{1}}^{+}$are tangent at a point in their boundaries or they meet at an interior point.

These situations cannot happen for a point of $\Gamma$ because $\widetilde{\psi}$ is differentiable and all tangent lines to $\Gamma$ do not pass through $p$. Then, since (3) is an elliptic equation, we have that $\Sigma_{t_{1}}^{-}$and $\Sigma_{t_{1}}^{+}$would be the same surface, which is a contradiction.

Remark 6. From Remark 5, the solution to the Plateau problem associated with a Jordan curve $\Gamma$ given by the above proposition does not depend on the selected conformal equivalence $G$ from $\mathbb{D}$ onto $\mathbb{I}_{\Gamma}$.

Moreover, if $\psi$ is an embedding, the hyperbolic normal to the immersion points towards $\mathcal{O}$. Otherwise, the hyperbolic normal would point upwards at the (Euclidean) highest point $p_{0}$ of the immersion, but the vertical straight half lines are hyperbolic geodesics and so $G\left(p_{0}\right)=\infty$ which contradicts the fact that $G(\mathbb{D}) \subset \mathbb{I}_{\Gamma}$.

First, we solve the Plateau problem at infinity when the Jordan curve is convex.

Theorem 5. Let $\Gamma$ be a convex Jordan curve on $\Pi \equiv\left\{x_{3}=0\right\} \subset \mathbb{R}^{3}$. Then for any $\varepsilon<0$ there exists a unique embedded solution to the Plateau problem for $\Gamma$ with hyperbolic normal pointing downwards at its (Euclidean) highest point.

Moreover if $-1 / 2<\varepsilon<0$, then there are exactly two embedded solutions to the Plateau problem for $\Gamma$.

Proof. Let us consider the bounded component $\operatorname{int}(\Gamma)$ of $\Pi \backslash \Gamma$ and a conformal equivalence $G$ from $\mathbb{D}$ onto $\mathbb{I}_{\Gamma}=\left\{x_{1}+i x_{2} \in \mathbb{C}:\left(x_{1}, x_{2}, 0\right) \in \operatorname{int}(\Gamma)\right\}$. Then, from 14] or [9, Corollary 3], we obtain $|\{G, z\}| \leq 2 /\left(1-|z|^{2}\right)^{2}<2(1-\varepsilon) /\left(-\varepsilon\left(1-|z|^{2}\right)^{2}\right)$, $\forall \varepsilon<0$. Therefore, from Proposition 1 the immersion $\psi$ associated with $\varepsilon$ and $G$ is a solution of the Plateau problem for $\Gamma$. 
Now, we shall prove that $\psi$ is an embedding. We observe that the induced metric $I$ of the immersion $\psi$ is conformal to its usual metric $I_{0}$ as an immersion into $\mathbb{R}^{3}$, in fact, from (33), $I_{0}=\psi_{3}^{2} I$, where $\psi_{3}>0$ denotes the third coordinate immersion. Hence, the Euclidean curvature $K_{0}$ of $\psi$ can be calculated as

$$
\psi_{3}^{2} K_{0}=K_{I}-\Delta^{I}\left(\log \psi_{3}\right)
$$

here $\Delta^{I}$ denotes the Laplacian associated with the induced metric $I$.

Since the Weierstrass data of the immersion $\psi$ are given by $h(z)=z / \sqrt{-\varepsilon}$ and $\omega=-1 / 2 \sqrt{-\varepsilon}\{G, z\} d z$, we obtain from (19), (23), (34) and by a straightforward computation of the above Laplacian that

$$
K_{I}=\frac{-16 \varepsilon}{\varepsilon^{2}|\{G, z\}|^{2}\left(1-|z|^{2}\right)^{4}-4(1-\varepsilon)^{2}}
$$

and

$$
K_{0}=\frac{-4 \varepsilon\left(4\left(1-|\mathcal{A}|^{2}\right)^{2}-|\{G, z\}|^{2}\left(1-|z|^{2}\right)^{4}\right)}{\left|G_{z}\right|^{2}\left(1-|z|^{2}\right)^{2}\left(4(1-\varepsilon)^{2}-\varepsilon^{2}\left(1-|z|^{2}\right)^{4}\right)} .
$$

Thus, by using [9] Corollary 3], one has $4\left(1-|\mathcal{A}|^{2}\right)^{2}-|\{G, z\}|^{2}\left(1-|z|^{2}\right)^{4} \geq 0$ and $\psi$ is an immersion with nonnegative Euclidean curvature and planar boundary, that is, $\psi$ is an embedding (see, for instance, 7 or $[27$ ).

From Remark 6 the hyperbolic normal of the immersion $\psi$ points downwards at its (Euclidean) highest point. Let us assume $\chi: S \longrightarrow \mathbb{H}^{3} \subset \mathbb{R}^{3}$ is another solution to the Plateau problem for a fixed $\varepsilon_{0}<0$ and the convex Jordan curve $\Gamma$, with hyperbolic normal pointing downwards at its highest point, or equivalently, pointing towards the enclosed Euclidean domain by $\mathrm{cl}(\chi(S)) \cup \operatorname{int}(\Gamma)$.

Without loss of generality we may assume the origin belongs to $\operatorname{int}(\Gamma)$. Let $C_{0}$ and $C_{2}$ be two circles on $\Pi$ centered at the origin and bounding a closed annulus $A$ in $\Pi$ containing $\Gamma$ in its interior. Choose $C_{0}$ and $C_{2}$ so that the totally umbilical BLW-surfaces (spherical caps, with hyperbolic normal pointing downwards) $S_{0}$ and $S_{2}$ associated with $\varepsilon_{0}$ and asymptotic boundary $C_{0}$ and $C_{2}$, respectively, satisfy that $S_{0}$ is below $\chi(S)$ and $\chi(S)$ is below $S_{2}$ (see Example 1).

Then, we consider $\Gamma_{t}, 0 \leq t \leq 2$, a foliation of the annulus $A$, such that $\Gamma_{t}$ is convex for all $t \in[0,2], \Gamma_{0}=C_{0}, \Gamma_{1}=\Gamma$ and $\Gamma_{2}=C_{2}$. Let $G_{t}$ be the conformal equivalence from $\mathbb{D}$ onto $\left\{x_{1}+i x_{2} \in \mathbb{C}:\left(x_{1}, x_{2}, 0\right) \in \operatorname{int}\left(\Gamma_{t}\right)\right\}$ such that $G_{t}(0)=0$ and $\left(G_{t}\right)_{z}(0)$ is a positive real number. Since $\Gamma_{t}$ is also convex we have $\left|\left\{G_{t}, z\right\}\right| \leq$ $2 /\left(1-|z|^{2}\right)^{2}$ (see [14) and we can consider, from Proposition 10 the BLW-surface $\psi_{t}: \mathbb{D} \longrightarrow \mathbb{H}^{3} \subset \mathbb{R}^{3}$ associated with $\varepsilon_{0}$ and hyperbolic Gauss map $G_{t}$. In particular, $\psi_{0}(\mathbb{D})=S_{0}, \psi_{1}(\mathbb{D})=\psi(\mathbb{D})$ and $\psi_{2}(\mathbb{D})=S_{2}$. Moreover, $\psi_{t}$ is an embedding as it was proved for $\psi$.

We want to show that $\chi(S)=\psi_{1}(\mathbb{D})$. First, we observe that if $t_{n} \in[0,2]$ and $\left\{t_{n}\right\}$ is a sequence converging to $t_{0}$, then $\left\{\widetilde{G_{t_{n}}}\right\}$ converges uniformly to $\widetilde{G_{t_{0}}}$ (see [8] Theorem II.5.2]), where $\widetilde{G_{t}}$ is the extension of $G_{t}$ to $\overline{\mathbb{D}}$.

Let us prove that if $0 \leq t<1$, then $\psi_{t}(\mathbb{D})$ is below $\chi(S)$. If $J=\{t \in[0,1$ : $\left.\psi_{t}(\mathbb{D}) \cap \chi(S) \neq \emptyset\right\}$ is nonempty, then we see that $J$ has a minimum; indeed we observe that given a sequence $\left\{t_{n}\right\} \subset J$ converging to the infimum $t_{0}$ of $J$, there would exist $p_{n} \in \mathbb{D}$ such that $\psi_{t_{n}}\left(p_{n}\right) \in \operatorname{cl}(\chi(S))$ and a subsequence $\left\{p_{m}\right\}$ would converge to a point $p_{0} \in \overline{\mathbb{D}}$ and, since $\left\{\psi_{t_{m}}\right\}$ converges uniformly to $\psi_{t_{0}}$, then $\psi_{t_{0}}\left(p_{0}\right) \in \operatorname{cl}(\chi(S))$. But, $p_{0} \notin \partial \mathbb{D}$ because of $\Gamma_{t_{0}} \cap \Gamma_{1}=\emptyset$, that is, $t_{0} \in J$.

Assume $J \neq \emptyset$; then, using the uniform convergence for any $t \in\left[0, t_{0}[\right.$, one has that $\psi_{t}$ and $\psi_{t_{0}}$ are below $\chi(S)$. Thus, since $\psi_{t_{0}}(\mathbb{D})$ and $\chi(S)$ are tangent at 
some point with the same hyperbolic normal and $\psi_{t_{0}}(\mathbb{D})$ is below $\chi(S)$, we have that $\psi_{t_{0}}(\mathbb{D})$ and $\chi(S)$ agree, but this is not possible because they have different asymptotic boundary.

Consequently, $J=\emptyset$ and using another time the uniform convergence one has $\psi_{1}(\mathbb{D})$ is below $\chi(S)$. Analogously, it can be shown that $\chi(S)$ is below $\psi_{1}(\mathbb{D})$ reasoning for the interval $[1,2]$ and that it yields $\psi_{1}(\mathbb{D})=\chi(S)$.

Let us now consider $\left.\varepsilon_{0} \in\right]-1 / 2,0[$. We want to prove that there exists another embedded solution to the Plateau problem for $\varepsilon_{0}$ and for the convex Jordan curve $\Gamma$. Let $\Gamma_{t}, 0 \leq t \leq 1$, be a foliation of the topological annulus bounded by the circle $C_{0}$ and $\Gamma$ in $\Pi$ such that $\Gamma_{t}$ is smooth for any $t \in\left[0,1\left[, \Gamma_{0}=C_{0}\right.\right.$ and $\Gamma_{1}=\Gamma$.

Let $f: \mathbb{R}^{3} \backslash\{(0,0,0)\} \longrightarrow \mathbb{R}^{3} \backslash\{(0,0,0)\}$ be the map given by

$$
f\left(x_{1}, x_{2}, x_{3}\right)=\left(\frac{x_{1}}{x_{1}^{2}+x_{2}^{2}+x_{3}^{2}}, \frac{-x_{2}}{x_{1}^{2}+x_{2}^{2}+x_{3}^{2}}, \frac{x_{3}}{x_{1}^{2}+x_{2}^{2}+x_{3}^{2}}\right),
$$

where $f$ is a preserving orientation hyperbolic isometry when it is restricted to $\mathbb{H}^{3}$.

Bearing in mind that the origin belongs to $\operatorname{int}\left(\Gamma_{t}\right)$ for any $t \in[0,1]$, we take the Jordan curves $\varphi_{t}=f\left(\Gamma_{t}\right) \subset \Pi$ (not necessarily convex). We also call $G_{t}$ to the conformal equivalence from $\mathbb{D}$ onto $\left\{x_{1}+i x_{2}:\left(x_{1}, x_{2}, 0\right) \in \operatorname{int}\left(\varphi_{t}\right)\right\}$ such that $G_{t}(0)=0$ and $\left(G_{t}\right)_{z}(0)$ is a positive real number. Then, from [13], one has $\left|\left\{G_{t}, z\right\}\right| \leq 6 /\left(1-|z|^{2}\right)^{2}$ and, using that $-1 / 2<\varepsilon_{0}$ and Proposition 11, the immersion $\psi_{t}: \mathbb{D} \longrightarrow \mathbb{H}^{3}$ associated with $\varepsilon_{0}$ is well defined and $\psi_{t}$ is a solution to the Plateau problem for the Jordan curve $\varphi_{t}$, or equivalently, $f \circ \psi_{t}$ is a solution to the Plateau problem for $\Gamma_{t}$ (since $f \circ f$ is the identity). Moreover, if $\psi_{t}$ is an embedding with hyperbolic normal pointing downwards at its highest point, then $f \circ \psi_{t}$ is an embedding with hyperbolic normal pointing upwards at its highest point because the origin is in $\operatorname{int}\left(\varphi_{t}\right)$.

We want to prove that $\psi_{1}$ is an embedding. If we define the interval $L=\{t \in$ $[0,1]: \psi_{s}$ is an embedding for all $\left.s \in[0, t]\right\}$, we know that $0 \in L$ and only need to prove that $L$ is open and closed in $[0,1]$.

We claim $L$ must be open. Otherwise, since $L$ is an interval, $L=\left[0, t_{0}\right]$ with $t_{0} \neq 1$ and there would exist a sequence $\left.\left\{t_{n}\right\} \subset\right] t_{0}, 1\left[\right.$ converging to $t_{0}$ such that $\psi_{t_{n}}$ is not an embedding. Hence, there would exist $p_{n}, q_{n} \in \mathbb{D}, p_{n} \neq q_{n}$, satisfying $\psi_{t_{n}}\left(p_{n}\right)=\psi_{t_{n}}\left(q_{n}\right)$. But a subsequence $\left\{p_{m}\right\}$ of $\left\{p_{n}\right\}$ must converge to a point $p_{0} \in \overline{\mathbb{D}}$ and a subsequence $\left\{q_{r}\right\}$ of $\left\{q_{m}\right\}$ converges to a point $q_{0} \in \overline{\mathbb{D}}$.

Reasoning as above, $\left\{\widetilde{\psi_{t_{r}}}\right\}$ converges uniformly to $\widetilde{\psi_{t_{0}}}$, where $\widetilde{\psi_{t}}$ denotes the differentiable extension of $\psi_{t}$ to $\overline{\mathbb{D}}$. In that way, $\widetilde{\psi_{t_{0}}}\left(p_{0}\right)=\widetilde{\psi_{t_{0}}}\left(q_{0}\right)$, but $\widetilde{\psi_{t_{0}}}$ is an embedding, that is, $p_{0}=q_{0}$.

From the uniform convergence, there exists a neighbourhood $U$ of $p_{0}=q_{0}$ in $\overline{\mathbb{D}}$ and $r_{0}$ such that $\widetilde{\psi_{t_{r}}}(U)$ is a graph on the tangent plane to the immersion $\widetilde{\psi_{t_{0}}}$ at $p_{0}=q_{0}$ for all $r \geq r_{0}$, which contradicts that $\widetilde{\psi_{t_{r}}}\left(p_{r}\right)=\widetilde{\psi_{t_{r}}}\left(q_{r}\right)$ with $p_{r} \neq q_{r}$ for $r$ big enough such that $p_{r}, q_{r} \in U$.

$L$ must be closed. We observe that the origin is a point in $\operatorname{int}\left(\varphi_{t}\right)$ for any $t \in[0,1]$ and the tangent line $l$ to $\varphi_{t}$ at a point $p$ does not pass through the origin, otherwise, we would obtain that the tangent line to $\Gamma_{t}=f\left(\varphi_{t}\right)$ at $f(p)$ is $f(l)=l$, which is a contradiction because $\Gamma_{t}$ is convex and the origin is in $\operatorname{int}\left(\Gamma_{t}\right)$.

Consequently, if the interval $L$ is not closed, then $L=\left[0, t_{0}[\right.$ and one has, from Proposition 1 , that the bounded Euclidean domain $\mathcal{O}_{t}$ enclosed by $\widetilde{\psi_{t}}(\overline{\mathbb{D}}) \cup \operatorname{int}\left(\Gamma_{t}\right)$ is starshaped from the origin for all $t \in L$. Again using the uniform convergence 
of $\widetilde{\psi_{t}}$ for $t \leq t_{0}$, the domain $\mathcal{O}_{t_{0}}$ enclosed by $\widetilde{\psi_{t_{0}}}(\overline{\mathbb{D}}) \cup \operatorname{int}\left(\Gamma_{t_{0}}\right)$ is also starshaped from the origin and then $\psi_{t_{0}}$ must be an embedding. Hence, $L=[0,1]$ and $\psi_{1}$ is an embedding.

The uniqueness of $f \circ \psi_{1}$ as the only embedding with asymptotic boundary $\Gamma$ and hyperbolic normal pointing upwards at its highest point can be proven in a similar way to the above case.

Remark 7. Notice that the Euclidean homotheties with center at a point of $\Pi$ are preserving orientation hyperbolic isometries when they are restricted to $\mathbb{H}^{3} \subset \mathbb{R}^{3}$ (see [24]) and then the uniqueness part of the above theorem could be proven in an easier way changing the above foliation and considering the foliation given by the image of $\psi(\mathbb{D})$ using the homotheties with center at a point in $\operatorname{int}(\Gamma)$.

Though this easier proof can be applied when $\operatorname{int}(\Gamma)$ is starshaped, it does not work for a general Jordan curve as it will be needed in Theorem 6.

Finally, we shall consider the case of a general Jordan curve $\Gamma$.

Theorem 6. Let $\Gamma$ be a Jordan curve on $\Pi \equiv\left\{x_{3}=0\right\} \subset \mathbb{R}^{3}$. Then for any $\varepsilon \in$ ] $-1 / 2,0$ [ there exist at least two solutions to the Plateau problem for $\Gamma$. Moreover, if $-1 / 4<\varepsilon<0$, then there only exist two embedded solutions to this Plateau problem.

Proof. Without loss of generality we can assume the origin belongs to $\operatorname{int}(\Gamma)$, thus reasoning as in Theorem 5, if we take $\varphi=f(\Gamma)$, where $f$ is given by (35), and

$$
\begin{array}{r}
G_{1}: \mathbb{D} \longrightarrow\left\{x_{1}+i x_{2}:\left(x_{1}, x_{2}, 0\right) \in \operatorname{int}(\Gamma)\right\}, \\
G_{2}: \mathbb{D} \longrightarrow\left\{x_{1}+i x_{2}:\left(x_{1}, x_{2}, 0\right) \in \operatorname{int}(\varphi)\right\}
\end{array}
$$

are two conformal equivalences, then, from $[13],\left|\left\{G_{i}, z\right\}\right| \leq 6 /\left(1-|z|^{2}\right)^{2}$, for $i=1,2$ and, using $\varepsilon \in]-1 / 2,0$ [ and Proposition 1] we can consider the immersions $\psi_{1}, \psi_{2}$ : $\mathbb{D} \longrightarrow \mathbb{H}^{3} \subset \mathbb{R}^{3}$ with associated hyperbolic Gauss map $G_{1}$ and $G_{2}$, respectively. Therefore, $\psi_{1}$ and $f \circ \psi_{2}$ are two solutions to the Plateau problem for $\Gamma$.

On the other hand, if $-1 / 4<\varepsilon<0$, then $\psi_{1}$ and $\psi_{2}$ must be embedded. In fact, we shall prove that $\psi_{1}$ is a graph on the plane $\Pi$ and analogously could be done for $\psi_{2}$.

From (29) and (32), the hyperbolic normal $\eta$ of $\psi_{1}$ is given by

$$
\eta=\left(-2 \varepsilon\left(G_{1}\right)_{z}\left(1-|z|^{2}\right) \frac{\overline{\mathcal{A}}}{\left(1-\varepsilon|\mathcal{A}|^{2}\right)^{2}},-\sqrt{-\varepsilon}\left|\left(G_{1}\right)_{z}\right|\left(1-|z|^{2}\right) \frac{1+\varepsilon|\mathcal{A}|^{2}}{\left(1-\varepsilon|\mathcal{A}|^{2}\right)^{2}}\right) .
$$

Also, since from (33) the induced metrics on $\psi_{1}$ from $\mathbb{R}^{3}$ and $\mathbb{H}^{3}$ are conformal, the standard Euclidean normal $N$ to $\psi_{1}$ is

$$
N=\left(2 \sqrt{-\varepsilon} \frac{\left(G_{1}\right)_{z}}{\left|\left(G_{1}\right)_{z}\right|} \frac{\overline{\mathcal{A}}}{1-\varepsilon|\mathcal{A}|^{2}},-\frac{1+\varepsilon|\mathcal{A}|^{2}}{1-\varepsilon|\mathcal{A}|^{2}}\right) .
$$

On the other hand, $|\mathcal{A}| \leq 2$ because of $\left|\left\{G_{1}, z\right\}\right| \leq 6 /\left(1-|z|^{2}\right)^{2}$ (see [16, Folgerung 2.3]) and the third coordinate of $N$ is negative. Consequently, the projection $p$ : $\psi_{1}(\mathbb{D}) \longrightarrow \operatorname{int}(\Gamma)$ given by $p\left(x_{1}, x_{2}, x_{3}\right)=\left(x_{1}, x_{2}\right)$ is a local diffeomorphism and it is proper, therefore, $p$ is a global diffeomorphism and $\psi_{1}(\mathbb{D})$ is a graph on the plane $\Pi$.

By considering a foliation $\Gamma_{t}, 0 \leq t \leq 2$, of an annulus $A$ centered at a point in $\operatorname{int}(\Gamma)$ such that $\Gamma_{0}$ is a small enough circle in $\operatorname{int}(\Gamma), \Gamma_{1}=\Gamma$ and $\Gamma_{2}$ is a big enough circle containing $\Gamma$ in its interior, one can prove the uniqueness part in a 
similar way to Theorem 5 , bearing in mind that any immersion $\psi_{t}$ associated with $\Gamma_{t}$ is an embedding.

Remark 8. A solution to the Plateau problem for a nonregular Jordan curve can be found in Example 2. Moreover, from Example 3, we can see that the condition of embeddedness in Theorems 5 and 6 is essential.

\section{REFERENCES}

[1] J. A. Aledo and J. A. Gálvez, Complete Surfaces in the Hyperbolic Space with a Constant Principal Curvature, to appear in Math. Nachr.

[2] R. L. Bryant, Surfaces of mean curvature one in hyperbolic space, Astérisque 154-155 (1987), 321-347.

[3] P. Collin, L. Hauswirth and H. Rosenberg, The geometry of finite topology Bryant surfaces, Ann. of Math. 153 (2001), 623-659. MR 2002j:53012

[4] J. B. Conway, Functions of one complex variable II, Springer-Verlag, New York, 1995. MR 96i:30001

[5] M. P. do Carmo and H. B. Lawson, On Alexandrov-Bernstein theorems in hyperbolic space, Duke Math. J. 50 (1983), 995-1003. MR 85f:53009

[6] J. A. Gálvez, A. Martínez and F. Milán, Flat surfaces in the hyperbolic 3-space, Math. Ann. 316 (2000), 419-435. MR 2002b:53013

[7] M. Ghomi, Gauss map, topology, and convexity of hypersurfaces with nonvanishing curvature, Topology 41 (2002), 107-117. MR 2002j:53013

[8] G. M. Goluzin, Geometric theory of functions of a complex variable, American Mathematical Society, Providence, R.I., 1969. MR 40:308

[9] R. Harmelin, Locally convex functions and the Schwarzian derivative, Israel J. Math. 67 (1989), 367-379. MR 90m:30014

[10] M. Kokubu, M. Umehara and K. Yamada, An elementary proof of Small's formula for null curves in $\operatorname{PSL}(2, \mathrm{C})$ and an analogue for Legendrian curves in $\operatorname{PSL}(2, \mathrm{C})$, Osaka J. Math. 40 (2003), 697-715.

[11] N. J. Korevaar, R. Kusner, W. H. Meeks and B. Solomon, Constant mean curvature surfaces in hyperbolic space, Amer. J. Math. 114 (1992), 1-43. MR 92k:53116

[12] G. Levitt and H. Rosenberg, Symmetry of constant mean curvature hypersurfaces in hyperbolic space, Duke Math. J. 52 (1985), 53-59. MR 86h:53063

[13] Z. Nehari, The Schwarzian derivative and schlicht functions, Bull. Am. Math. Soc. 55 (1949), 545-551. MR 10:696e

[14] Z. Nehari, A property of convex conformal maps, J. Analyse Math. 30 (1976), 390-393. MR 55:12901

[15] F. Pacard and F. Pimentel, Attaching handles to Bryant surfaces, preprint (available at http://arXiv.org/math.DG/0112224).

[16] C. Pommerenke, Linear-invariante Familien analytischer Funktionen I, Math. Ann. 155 (1964), 108-154. MR 29:6007

[17] H. Rosenberg and R. Sa Earp, The Geometry of Properly Embedded Special Surfaces in $\mathbf{R}^{3}$; e.g., Surfaces Satisfying $a H+b K=1$, where $a$ and $b$ are Positive, Duke Math. J. 73 (1994), 291-306. MR 95b:53010

[18] H. Rosenberg and J. Spruck, On the existence of convex hypersurfaces of constant Gauss curvature in hyperbolic space, J. Differential Geom. 40 (1994), 379-409. MR 95g:53007

[19] R. Sa Earp and E. Toubiana, Sur les surfaces de Weingarten spéciales de type minimal, Bol. Soc. Brasil. Mat. 26 (1995), 129-148. MR 96j:53006

[20] R. Sa Earp and E. Toubiana, Classification des surfaces de type Delaunay, Amer. J. Math. 121 (1999), 671-700. MR 2001b:53004

[21] R. Sa Earp and E. Toubiana, Symmetry of Properly Embedded Special Weingarten Surfaces in $\mathbb{H}^{3}$, Trans. Am. Math. Soc. 351 (1999), 4693-4711. MR 2000c:53007

[22] W. Rossman, M. Umehara and K. Yamada, Mean curvature 1 surfaces in hyperbolic 3-space with low total curvature I, preprint (available at http://arXiv.org/math.DG/0008015).

[23] A. J. Small, Surfaces of constant mean curvature 1 in $\mathrm{H}^{3}$ and algebraic curves on a quadric, P. Am. Math. Soc. 122 (1994), 1211-1220. MR 95b:53011 
[24] M. Spivak, A comprehensive introduction to Differential Geometry, Vol. 4, Publish or Perish, Inc., Berkeley, 1979. MR 82g:53003d

[25] M. Umehara and K. Yamada, Complete surfaces of constant mean curvature 1 in the hyperbolic 3-space, Ann. of Math. 137 (1993), 611-638. MR 94c:53015

[26] M. Umehara and K. Yamada, A parametrization of the Weierstrass formulae and perturbation of complete minimal surfaces in $\mathbf{R}^{3}$ into the hyperbolic 3-space, J. Reine Angew. Math. 432 (1992), 93-116. MR 94e:54004

[27] J. van Heijenoort, On locally convex manifolds, Comm. Pure Appl. Math. 5 (1952), 223-242. MR 14:577b

Departamento de Geometría y Topología, Facultad de Ciencias, Universidad de Granada, 18071 Granada, Spain

E-mail address: jagalvez@ugr.es

Departamento de Geometría y Topología, Facultad de Ciencias, Universidad de Granada, 18071 Granada, Spain

E-mail address: amartine@ugr.es

Departamento de Geometría y Topología, Facultad de Ciencias, Universidad de Granada, 18071 Granada, Spain

E-mail address: milan@ugr.es 